\title{
NORMAL-COORDINATE STRUCTURAL DECOMPOSITION \\ AND THE VIBRONIC SPECTRA OF PORPHYRINS
}

\author{
John A. Shelnutt \\ Nanomolecular Materials and Interfaces Department \\ 3 \\ Sandia National Laboratories \\ Albuquerque, NM 87185-1349 \\ RECEIVED \\ NOV 291999 \\ OSTI \\ and \\ Department of Chemistry \\ University of New Mexico \\ Albuquerque, NM 87131
}

For submission to: J. Porphyrins Phthalocyanines

September 8, 1999

Sandia is a multiprogram laboratory operated by Sandia Corporation, a Lockheed Martin Company, for the United States Department of Energy under Contract DE-AC04-94AL85000. 


\section{DISCLAIMER}

This report was prepared as an account of work sponsored by an agency of the United States Government. Neither the United States Government nor any agency thereof, nor any of their employees, make any warranty, express or implied, or assumes any legal liability or responsibility for the accuracy, completeness, or usefulness of any information, apparatus, product, or process disclosed, or represents that its use would not infringe privately owned rights. Reference herein to any specific commercial product, process, or service by trade name, trademark, manufacturer, or otherwise does not necessarily constitute or imply its endorsement, recommendation, or favoring by the United States Government or any agency thereof. The views and opinions of authors expressed herein do not necessarily state or reflect those of the United States Government or any agency thereof. 


\section{DISCLAIMER}

Portions of this document may be illegible in electronic image products. Images are produced from the best available original document. 


\begin{abstract}
The connection is made between the normal-coordinate structural decomposition (NSD) and the vibionic molecular states and spectra of porphyrins. NSD is a procedure that provides a description of the distortion of a porphyrin from a $D_{4 h}$ symmetric reference structure in terms of equivalent displacements along the normal coordinates. Expressions for the optical absorption spectra with vibrational structure are developed with these NSD-determined deformations as parameters, and the expressions are applied to the UV-visible absorption spectra porphyrins.
\end{abstract}




\section{INTRODUCTION}

Normal-coordinate structural decomposition (NSD)\{Jentzen, 1998 \#6367; Jentzen, 1997 $\# 610$ \} has become a powerful tool for the analysis of porphyrin structures, especially for quantifying out-of-plane distortion of the porphyrin macrocycle in heme proteins. \{Jentzen, 1998 $\# 6367$; Jentzen, $1997 \# 610$; Shelnutt, $1998 \# 127$ \} The NSD analysis of the structure of a porphyrin macrocycle determines the deformations along the normal coordinates that optimally describe the distortion of the porphyrin molecule from of a square-planar reference structure. The description of the macrocycle distortion in terms of the normal coordinates provides a uniquely useful representation of the conformation given in terms of the vibrational modes and energies of the molecule. One outcome of the NSD analysis is that the description of the porphyrin distortion is greatly simplified. The simplification occurs because only a few displacements along the lowest-frequency vibration modes are required to accurately depict the structure. These lowest-frequency modes represent the most flexible ways for the molecule to distort, and these modes usually exhibit the largest deformations. The simple NSD description is an alternative to listing, for example, the out-of-plane displacements of all 24 atoms of the macrocycle. The latter description is not particularly informative, and even if the distortion is along only one of the normal modes one still has to give all 24 out-of-plane displacements.

Given the unique relationship between the molecular vibrations and the normal coordinates, one would expect that vibrational spectra might have a strong dependence on the normal-coordinate displacements determined in the NSD analysis. Certainly, when the groundstate distortion lowers the symmetry of the porphyrin from $\mathrm{D}_{4 \mathrm{~h}}$, vibrational modes of symmetries not normally Raman or IR active will be observed. Similarly, the vibrational structure in the 
electronic absorption bands of porphyrins is altered by the macrocyclic distortion, and expressions for the absorbance of the $Q$ and $B$ (Soret) bands, including the vibrational satellites, are easily obtained in the weak vibro-electronic (vibronic) coupling limit. Similar expressions for the Raman excitation spectra (RES) also follow from the analysis. 


\section{The crude Born-Oppenheimer approximation and vibronic coupling}

The Born-Oppenheimer approximation provides a simplification of the molecular Hamiltonian based on the large difference in nuclear and electronic masses. Electrons are light and move much faster than the nuclei, thus we can effectively consider the nuclei fixed and calculate the motion of the electrons in the Coulomb field of the nuclei.

Consider a molecule consisting of $N$ electrons and $P$ nuclei with charges $Z_{p}$ and masses $M_{p}$.

The Hamiltonian for this system is

$$
\begin{gathered}
H=-\frac{\hbar^{2}}{2 m_{e}} \sum_{i=1}^{N} \nabla_{i}^{2}-\frac{\hbar^{2}}{2} \sum_{p=1}^{P}\left(\frac{1}{M_{p}}\right) \nabla_{p}^{2}-\sum_{i=1}^{N} \sum_{p=1}^{P} \frac{Z_{p} e^{2}}{r_{p i}}+\sum_{p>q}^{P} \frac{Z_{p} Z_{q} e^{2}}{R_{p q}}+\sum_{i>j}^{N} \frac{e^{2}}{r_{i j}} \\
=T_{e}+T_{N}+V(N-e)+V(N-N)+V(e-e),
\end{gathered}
$$

which can be rearranged as

$$
H=\left[-\frac{\hbar^{2}}{2 m_{e}} \sum_{i=1}^{N} \nabla_{i}^{2}-\sum_{p}^{p} \sum_{i}^{N} \frac{Z_{p} e^{2}}{r_{p i}}+\sum_{i>j}^{N} \frac{e^{2}}{r_{i j}}\right]+\left[-\frac{\hbar^{2}}{2} \sum_{p=1}^{P}\left(\frac{1}{M_{p}}\right) \nabla_{p}^{2}+\sum_{p>q}^{P} \frac{Z_{p} Z_{q} e^{2}}{R_{p q}}\right],
$$

where the terms in the first brackets include both electronic and nuclear coordinates and those in the last set of brackets are purely nuclear. The relative distances $r_{p i}, r_{i j}$, and $R_{p q}$ are the distance between the $p^{\text {th }}$ nucleus and the $i^{\text {th }}$ electron, the distance between the $i^{\text {th }}$ and $j^{\text {th }}$ electrons, and the distance between the $p^{\text {th }}$ and $q^{\text {th }}$ nuclei, respectively.

Starting with the crude Born-Oppenheimer approximation, we assume solutions of the form 


$$
\psi\left(\vec{r}_{i}, \vec{R}_{p}\right)=\phi\left(\vec{r}_{i}\right) \chi\left(\vec{R}_{p}\right)
$$

giving a complete separation of the electronic and nuclear motions. $\phi\left(\vec{r}_{i}\right)$ is a function of the electronic coordinates $\vec{r}_{i}$, which are related to the relative distances as indicated in Figure 1 for $\vec{r}_{p i}$. Similarly, $\chi\left(\vec{R}_{p}\right)$ is a function of only the nuclear coordinates $\vec{R}_{p}$ for each of the nuclei $p$. Substituting these molecular wavefunctions into the time-independent Schrödinger equation,

$$
H \psi=E \psi,
$$

we get

$$
\begin{gathered}
\chi\left(\vec{R}_{p}\right)\left(-\frac{\hbar^{2}}{2 m_{e}} \sum_{i=1}^{N} \nabla_{i}^{2}\right) \phi\left(\vec{r}_{i}\right)-\sum_{q=1}^{P} \sum_{i=1}^{N} \frac{Z_{q} e^{2}}{r_{q i}} \chi\left(\vec{R}_{p}\right) \phi\left(\vec{r}_{i}\right)+\chi\left(\vec{R}_{p}\right) \sum_{i>j} \frac{e^{2}}{r_{i j}} \phi\left(\vec{r}_{i}\right) \\
+\phi\left(\vec{r}_{i}\right)\left(-\frac{\hbar^{2}}{2} \sum_{q=1}^{P} \frac{1}{M_{q}} \nabla_{q}^{2}+\sum_{q>p}^{P} \frac{Z_{q} Z_{p} e^{2}}{R_{p q}}\right) \chi\left(\vec{R}_{p}\right)=E \chi\left(\vec{R}_{p}\right) \phi\left(\vec{r}_{i}\right) .
\end{gathered}
$$

Except for the second term on the left-hand side of the equation, the nuclear and electronic motions are separable.

The nuclear coordinate dependence comes in entirely through the terms,

$$
-\sum_{q=1}^{P} \sum_{n=1}^{N} \frac{Z_{q} e^{2}}{r_{q i}}+\sum_{q>p}^{P} \frac{Z_{q} Z_{p} e^{2}}{R_{p q}} .
$$

These potential energy terms contain all of the nuclear coordinate dependence of the Hamiltonian, and they can be expanded in a Taylor series about some zero point, which is usually taken to be the equilibrium geometry of the molecule in the ground state. That is, the full electronic part of the Hamiltonian can be written as 


$$
H^{e}=H_{0}^{e}\left(q_{i}, Q=0\right)+\sum_{K=1}^{3 P} Q_{K}\left(\frac{\partial H^{e}}{\partial Q_{K}}\right)_{Q=0}+\frac{1}{2} \sum_{K, L=1}^{3 P} Q_{k} Q_{L}\left(\frac{\partial^{2} H^{e}}{\partial Q_{K} \partial Q_{L}}\right)_{Q=0}+\cdots
$$

where we have made a coordinate transformation to the set of normal coordinates of the ground electronic state $g$ that are zero at the equilibrium positions of the nuclei $(Q=0)$. The normal coordinates generally involve concerted motions of many of the nuclei of the macrocycle. $H^{e}$ is the full Hamiltonian of the molecule missing only the term that is the kinetic energy of the nuclei,

$$
-\frac{\hbar^{2}}{2} \sum_{K=1}^{3 P} \frac{1}{\mu_{K}} \frac{\partial^{2}}{\partial Q_{K}^{2}}
$$

as expressed in the new coordinates, where $\mu_{K}$ is the reduced mass for the coordinate $Q_{K}$. We can also show that both the kinetic and potential energies can be written in this diagonal form when the $Q_{K}$ are the normal coordinates, though this is not obvious. Mixing the coordinate systems since we will ultimately solve parts of the problem independently, the full molecular Hamiltonian can be written as

$$
\begin{aligned}
H=[ & \left.-\frac{\hbar^{2}}{2 m_{e}} \sum_{i} \nabla_{i}^{2}-\sum_{p} \sum_{i} \frac{Z_{p} e^{2}}{r_{0_{p i}}}+\sum_{i>j} \frac{e^{2}}{r_{i j}}+\sum_{p>q} \frac{Z_{p} Z_{q} e^{2}}{R_{0_{p q}}}\right] \\
& +\left[\sum_{K=1}^{3 P-6} Q_{K}\left(\frac{\partial H}{\partial Q_{K}}\right)_{Q=0}+\frac{1}{2} \sum_{L, K=1}^{3 P-6} Q_{K} Q_{L}\left(\frac{\partial^{2} H}{\partial Q_{K} \partial Q_{L}}\right)_{Q=0}+\cdots\right]+\left[-\frac{\hbar^{2}}{2} \sum_{K=1}^{3 P-6} \frac{1}{\mu_{K}} \frac{\partial^{2}}{\partial Q_{K}^{2}}\right] .
\end{aligned}
$$

In this expression, we have separated the Hamiltonian into three terms.

\section{The $0^{\text {th }}$ order electronic states}

The first term, $H_{0}^{e}\left(q_{i}, Q=0\right)$, has the nuclei fixed as indicated by the 0 of $r_{p i}$ and $R_{0_{p q}}$ 
in the denominators, and only the electrons move. This term gives the usual molecular orbital problem for a single-point calculation (with $\mathrm{CI}$ ). We assume that we can solve this electronic part of the problem at least approximately. The solutions of the electronic Schrödinger equation with clamped nuclei, i.e., the states $\left|l_{v}\left(\vec{r}_{i}\right)\right\rangle$ and energies $E_{l_{v}}^{0}$, satisfy

$$
H_{0}^{e}\left|l_{v}\left(\vec{r}_{i}\right)\right\rangle=E_{l}^{0}\left|l_{v}\left(\vec{r}_{i}\right)\right\rangle
$$

Here, $v$ is a subscript that runs over all degenerate states with energy $E_{l}^{0}$.

The second term of Eq (8) couples the electronic and nuclear motion and we treat it as a perurbation. The third term is the kinetic energy of the nuclei.

\section{The $0^{\text {th }}$ order vibrational states}

We now consider the vibrational motion problem, which we solve in terms of the normal coordinates for the electronic ground electronic state, $|g\rangle$, which we just obtained formally in solving Eq (9). Thus, $|g\rangle$ satisfies

$$
H_{0}^{e}|g\rangle=E_{g}^{0}|g\rangle
$$

Now, in the crude Born-Oppenheimer approximation, the molecular states are both $Q_{K}$ and $q_{i}$ dependent and are given by product states composed of an electronic wavefunction and a nuclear wavefunction, thus, for the molecular ground states,

$$
\psi\left(\vec{r}_{i}, \vec{R}_{p}\right)=|g\rangle\left|\chi\left(Q_{K}\right)\right\rangle
$$

If we let the full molecular Hamiltonian, $H=H^{e}+T_{N}$, operate on this $0^{\text {th }}$ order molecular state, we obtain 


$$
H \psi\left(\vec{r}_{i}, \vec{R}_{p}\right)=H|g\rangle\left|\chi_{\vec{v}}\left(Q_{K}\right)\right\rangle=\left(H^{e}+T_{N}\right)|g\rangle\left|\chi_{\vec{v}}\right\rangle,
$$

where $\vec{v}$ is a vector of vibrational quantum numbers of dimension $3 P-6$. If we now multiply on the left by the electronic state $\langle g|$ and integrate over the electronic coordinates, we have for the right side of equation (12),

$$
\left\langle\left\langle g\left|H_{i}^{e}\right| g\right\rangle+T_{N}\langle g \mid g\rangle\right)\left|\chi_{\vec{v}}\right\rangle
$$

where,

$$
\left\langle g\left|H^{e}\right| g\right\rangle=\left\langle g\left|H_{0}^{e}\right| g\right\rangle+\sum_{K=1}^{3 N} Q_{K}\left\langle g\left|\left(\frac{\partial H^{e}}{\partial Q_{K}}\right)_{0}\right| g\right\rangle+\frac{1}{2} \sum_{K, L=1}^{3 N} Q_{K} Q_{L}\left\langle g\left(\frac{\partial^{2} H^{e}}{\partial Q_{K} \partial Q_{L}}\right)_{0} \mid g\right\rangle+\cdots
$$

Truncating the series, we have the approximate expression,

$$
\left\langle g\left|H^{e}\right| g\right\rangle \approx E_{g}^{0}+\sum_{K} Q_{K}\left\langle g\left|\left(\frac{\partial H^{e}}{\partial Q_{K}}\right)_{0}\right| g\right\rangle+\frac{1}{2} \sum_{K} \sum_{L} Q_{K} Q_{L}\left\langle g\left|\left(\frac{\partial^{2} H^{e}}{\partial Q_{K} \partial Q_{L}}\right)_{0}\right| g\right\rangle .
$$

So we have,

$$
\begin{aligned}
& \left\langle\left\langle g\left|H^{e}\right| g\right\rangle+T_{N}\langle g \mid g\rangle\right)\left|\chi_{n}\right\rangle= \\
& {\left[E_{g}^{0}+\sum_{K} Q_{K}\left\langle g\left|\left(\frac{\partial H^{e}}{\partial Q_{K}}\right)_{0}\right| g\right\rangle+\frac{1}{2} \sum_{K} \sum_{L} Q_{K} Q_{L}\left\langle g\left(\frac{\partial^{2} H^{e}}{\partial Q_{K} \partial Q_{L}}\right)_{0} \mid g\right\rangle+T_{N}\right]\left|\chi_{n}\right\rangle}
\end{aligned}
$$

Usually we proceed by assuming that the Taylor expansion is about the equilibrium positions of the nuclei, and thus, the linear terms must vanish. That is, the slope at the bottom of the potential well in which the nuclei move must be zero. Said another way "expanding about the equilibrium position" means there can be no force on the nuclei at $Q_{1}=0, \cdots, Q_{K}=0, \cdots$. Defining the zero point in this way is not necessary however, and we will relax this condition 
later in the theory development. Alternatively, we might expand instead about the reference $D_{4 h}$ porphyrin macrocycle of the NSD analysis. Porphyrins that exhibit distortions from the reference structure will not have $\left\langle g\left|\left(\frac{\partial H^{e}}{\partial Q_{L}}\right)_{0}\right| g\right\rangle=0$ for all normal coordinates, $Q_{L}$. These linear terms of the ground state are related to the displacements along the normal coordinates measured for a distorted porphyrin macrocycle by the NSD analysis of its structure. For now, we can neglect these terms and then include them later as part of the vibronic perturbation Hamiltonian for distorted porphyrins.

The quadratic term is diagonal in the normal coordinate system, ${ }^{2}$ and we write this term for the ground electronic state as

$$
\frac{1}{2} \sum_{K, L} Q_{K} Q_{L}\left\langle g\left|\left(\frac{\partial^{2} H^{e}}{\partial Q_{K} \partial Q_{L}}\right)_{0}\right| g\right\rangle=\frac{1}{2} \sum_{K} Q_{K}^{2} f_{K},
$$

where the $f_{K}$ are the force constants of a harmonic oscillator,

$$
f_{K}=\left\langle g\left|\left(\frac{\partial^{2} H^{e}}{\partial Q_{K}^{2}}\right)_{0}\right| g\right\rangle
$$

Thus, finally we have for the ground electronic state,

$$
\left\langle\left\langle g\left|H^{e}\right| g\right\rangle+T_{N}\langle g \mid g\rangle\right)\left|\chi_{\vec{v}}\right\rangle=\left[T_{N}+E_{g}^{0}+\frac{1}{2} \sum_{K} Q_{K}^{2} f_{K}\right]\left|\chi_{\vec{v}}\right\rangle .
$$

The term in square brackets is the nuclear Hamiltonian for the ground state. We now see from Eq. (17) that the potential in which the nuclei move in the ground electronic state is the harmonic electronic potential energy, 


$$
E_{g}\left(Q_{K}\right)=E_{g}^{0}+\frac{1}{2} \sum_{K} Q_{K}^{2} f_{K}
$$

and the Schrödinger equation for the vibrational states $\left|\chi_{\bar{\nu}}\left(Q_{K}\right)\right\rangle$ of the ground electronic state is that for a collection of $3 P-6$ independent harmonic oscillators,

$$
\left[\sum_{K}\left(-\frac{\hbar^{2}}{2 \mu_{K}} \frac{\partial^{2}}{\partial Q_{K}^{2}}+\frac{1}{2} f_{K} Q_{K}^{2}\right)+E_{g}^{0}\right]\left|\chi_{\vec{v}}\right\rangle=E_{g, \vec{v}}^{0}\left|\chi_{\vec{v}}\right\rangle .
$$

(Here we have ignored the rotational and translation motion.) The oscillators are independent because of the diagonal form.

The solutions of the independent harmonic oscillators problem are well known; the states $\left|\chi_{\bar{v}}\right\rangle$ are products of $3 P-6$ Hermite polynomial functions of the normal coordinates with energies given by

$$
E_{g \vec{v}}=E_{g}^{0}+\sum_{K}\left(v_{K}+\frac{1}{2}\right) \hbar \Omega_{K},
$$

where the vibrational angular frequency is

$$
\Omega_{K}=\sqrt{\frac{f_{K}}{\mu_{K}}} .
$$

The vector $\vec{v}$ represents $3 P-6$ integers giving the excitation level of each vibration,

$$
\left|\chi_{\vec{v}}\left(Q_{K}\right)\right\rangle=\left|v_{K} \cdots\right\rangle=\left|v_{1} \cdots, v_{K}, \cdots v_{3 P-6}\right\rangle=\left|v_{1}\right\rangle\left|v_{2}\right\rangle \cdots\left|v_{K}\right\rangle \cdots\left|v_{3 P-6}\right\rangle,
$$

where we have dropped $\chi$ and labeled the vibrational state with the quantum numbers $\bar{v}$. The multimode vibrational state is simply a product of the Hermite polynomial states for each of the $3 P-6$ oscillators. 


\section{The $0^{\text {th }}$ order molecular states}

Although these vibrational states are energy eigenstates only for the ground electronic state, nonetheless they form a basis (i.e., they span the space) of the nuclear coordinates. Thus, we can take as our $0^{\text {th }}$ order molecular states,

$$
|\psi\rangle=\left|l_{v}\right\rangle|\vec{v}\rangle,
$$

where the vibrational states are those of the ground state, and linear combinations of these states will provide improved first order solutions of the full molecular Hamiltonian, $H=H_{0}+H^{\prime}$. The $0^{\text {th }}$ order states satisfy the $0^{\text {th }}$ order Hamiltonian $H_{0}$, which has the form,

$$
H_{0}=\left[-\frac{\hbar^{2}}{2 m_{e}} \sum_{i=1} \nabla_{i}^{2}-\sum_{p} \sum_{i} \frac{Z_{p} e^{2}}{r_{o_{p i}}}+\sum_{i>j} \frac{e^{2}}{r_{i j}}+\sum_{p>q} \frac{Z_{p} Z_{q} e^{2}}{R_{o_{p q}}}\right]+\left[\frac{1}{2} \sum_{K} Q_{K}^{2} f_{K}-\frac{\hbar^{2}}{2} \sum_{K} \frac{1}{\mu_{K}} \frac{\partial^{2}}{\partial Q_{K}^{2}}\right]
$$

where the first bracket represents the $0^{\text {th }}$ order electronic Hamiltonian and the second term is the $0^{\text {th }}$ order vibrational Hamiltonian.

The Hamiltonian $H^{\prime}$ describing the interaction between the $0^{\text {th }}$ order states is just $H^{\prime}=H-H_{0}$, where from $\mathrm{Eq}(8)$,

$$
\begin{aligned}
H=[- & \left.\frac{\hbar^{2}}{2 m_{e}} \sum_{i} \nabla_{i}^{2}-\sum_{p} \sum_{i} \frac{Z_{p} e^{2}}{r_{o_{p i}}}+\sum_{i>j} \frac{e^{2}}{r_{i j}}+\sum_{p>q} \frac{Z_{p} Z_{q} e^{2}}{R_{o_{p q}}}\right] \\
& +\left[\sum_{K} Q_{K}\left(\frac{\partial H}{\partial Q_{K}}\right)_{0}+\frac{1}{2} \sum_{K, L} Q_{K} Q_{L}\left(\frac{\partial H}{\partial Q_{K} \partial Q_{L}}\right)_{0}-\frac{\hbar^{2}}{2} \sum_{K} \frac{1}{\mu_{K}} \frac{\partial^{2}}{\partial Q_{K}^{2}}\right]
\end{aligned}
$$

Subtracting $H_{0}$ from $H$, we get

$$
H^{\prime}=\sum_{K} Q_{K}\left(\frac{\partial H}{\partial Q_{K}}\right)_{0}+\frac{1}{2} \sum_{K, L}\left[Q_{K} Q_{L}\left(\frac{\partial H}{\partial Q_{K} \partial Q_{L}}\right)_{0}-f_{K} \delta_{K, L}\right]
$$

$H^{\prime}$ is the vibronic interaction that mixes the $0^{\text {th }}$ order product states of $\mathrm{Eq}(23)$ and couples the 
$0^{\text {th }}$ order electronic states with the $0^{\text {th }}$ order vibrational states. We can now use this vibronic interaction Hamiltonian to obtain perturbative solutions to the vibronic coupling problem.

\section{The $1^{\text {st }}$ order vibronic molecular states}

From time-dependent perturbation theory, the eigenstates to first order in the interaction, $H^{\prime}$, are as shown below: ${ }^{3}$

$$
\begin{aligned}
|l\rangle=\left|l_{v}\right\rangle|\vec{v}\rangle-\sum_{j, \alpha} \sum_{\vec{u}} \sum_{K=1}^{3 N-6} \frac{\left\langle j_{\alpha}\left|\left(\frac{\partial H^{\prime}}{\partial Q_{K}}\right)_{0}\right| l_{v}\right\rangle\left\langle\vec{u}\left|Q_{K}\right| \vec{v}\right\rangle}{E_{l_{v} \vec{v}}-E_{j_{\alpha} \vec{u}}}\left|j_{\alpha}\right\rangle|\vec{u}\rangle f_{j_{\alpha} \vec{u}, l_{v} \vec{v}}\left(t_{0}\right) \\
-\sum_{j, \alpha} \sum_{\vec{u}} \sum_{K, L=1}^{3 N-6} \frac{\left\langle j_{\alpha}\left|\left(\frac{\partial^{2} H^{\prime}}{\partial Q_{K} \partial Q_{L}}\right)_{0}\right| l_{v}\right\rangle\left\langle\bar{u}\left|Q_{K} Q_{L}\right| \vec{v}\right\rangle}{E_{l_{v} \vec{v}}-E_{j_{\alpha} \vec{u}}}\left|j_{\alpha}\right\rangle|\vec{u}\rangle f_{j_{\alpha} \vec{u}, l_{v} \vec{v}}\left(t_{0}\right)
\end{aligned}
$$

+ high order perturbation terms and high order Taylor series expansion terms, where $f_{j_{\alpha} \vec{u}, l_{v} \vec{v}}\left(t_{0}\right)=1-e^{i\left(E_{j}^{0}-E_{l}^{0}\right)\left(t_{0}-t_{0}^{\prime}\right) / \hbar}$. Notice that the first order states involve the energies only to $0^{\text {th }}$ order. The molecular states, $|l\rangle$, are the approximate vibronic molecular states that we will use to introduce vibrational structure into our equations for the different types of electronic and vibrational spectra.

\section{Evaluation of the vibrational matrix elements}

Our next step is to evaluate the vibrational matrix elements in the above expression for the approximate vibronic states. The vibrational matrix elements can be evaluated by remembering that for a harmonic oscillator the coordinate can be expressed in terms of the creation and annihilation operators as 


$$
Q_{K}=\frac{1}{\sqrt{2} f_{K}}\left(A_{K}+A_{K}^{\dagger}\right)
$$

Thus,

$$
\begin{gathered}
\left\langle\vec{u}\left|Q_{K}\right| \vec{v}\right\rangle=\left\langle u_{1}, \cdots, u_{3 P-6}\left|Q_{K}\right| v_{1}, \cdots, v_{3 P-6}\right\rangle=\frac{1}{\sqrt{2 f_{K}}}\left\langle u_{1}\left|\cdots\left\langle u_{3 P-6}\left|\left(A_{K}+A_{k}^{\dagger}\right)\right| v_{3 P-6}\right\rangle \cdots\right| v_{1}\right\rangle \\
\left\langle\vec{u}\left|Q_{K}\right| \vec{v}\right\rangle= \begin{cases}\frac{1}{\sqrt{2 f_{K}}}\left\langle v_{K}-1\left|A_{K}\right| v_{K}\right\rangle, & \text { if } u_{K}=v_{K}-1 \\
\frac{1}{\sqrt{2 f_{K}}}\left\langle v_{K}+1\left|A_{K}^{\dagger}\right| v_{K}\right\rangle, & \text { if } u_{K}=v_{K}+1\end{cases}
\end{gathered}
$$

and all other $u_{L}=v_{L}$ for $L \neq K$. Now, using the relationships,

$$
A_{K}\left|v_{K}\right\rangle=\left(v_{K} \hbar \Omega_{K}\right)^{1 / 2}\left|v_{K}-1\right\rangle
$$

and

$$
A_{K}^{\dagger}\left|v_{K}\right\rangle=\left[\left(v_{K}+1\right) \hbar \Omega_{K}\right]^{1 / 2}\left|v_{K}+1\right\rangle
$$

we have

$$
\left\langle\vec{u}\left|Q_{K}\right| \vec{v}\right\rangle= \begin{cases}\frac{1}{\sqrt{2 f_{K}}}\left[v_{K} \hbar \Omega_{K}\right]^{1 / 2}, & u_{K}=v_{K}-1 \text { and } u_{L}=v_{L}, \forall L \neq K \\ \frac{1}{\sqrt{2 f_{K}}}\left[\left(v_{K}+1\right) \hbar \Omega_{K}\right]^{1 / 2}, & u_{K}=v_{K}+1 \text { and } u_{L}=v_{L}, \forall L \neq K \\ 0, & \text { otherwise. }\end{cases}
$$

Similarly, the bilinear matrix elements are

$$
\left\langle\bar{u}\left|Q_{K} Q_{L}\right| \vec{v}\right\rangle=\left\langle\vec{u}\left|\left(A_{K}+A_{K}^{\dagger}\right)\left(A_{L}+A_{L}^{\dagger}\right)\right| \vec{v}\right\rangle=\left\langle\bar{u}\left|A_{K} A_{L}^{\dagger}\right| \bar{v}\right\rangle+\left\langle\bar{u}\left|A_{K}^{\dagger} A_{L}\right| \vec{v}\right\rangle+\left\langle\bar{u}\left|A_{K} A_{L}\right| \vec{v}\right\rangle,
$$

and the matrix elements that survive are 


$$
\left\langle\vec{u}\left|Q_{K} Q_{L}\right| \vec{v}\right\rangle=\frac{\hbar}{2 \sqrt{\mu_{K} \Omega_{K} \mu_{L} \Omega_{L}}} \begin{cases}\sqrt{v_{K}\left(v_{L}+1\right)}, & u_{K}=v_{K}-1, u_{L}=v_{L}+1 \\ \sqrt{\left(v_{K}+1\right) v_{L}}, & u_{K}=v_{K}+1, u_{L}=v_{L}-1 \\ \sqrt{v_{K} v_{L}}, & u_{K}=v_{K}-1, u_{L}=v_{L}-1 \\ \sqrt{\left(v_{K}+1\right)\left(v_{L}+1\right)}, & u_{K}=v_{K}+1, u_{L}=v_{L}+1,\end{cases}
$$

and for $K=L$ this reduces to

$$
\left\langle\vec{u}\left|Q_{K}^{2}\right| \vec{v}\right\rangle=\frac{\hbar}{2 \mu_{K} \Omega_{K}} \begin{cases}\sqrt{v_{K}\left(v_{L}+1\right)}, & u_{K}=v_{K} \\ v_{K}, & u_{K}=v_{K}-2 \\ \left(v_{K}+1\right), & u_{K}=v_{K}+2\end{cases}
$$

Because the matrix elements like $\langle\vec{u} \mid \vec{v}\rangle$ and $\left\langle\vec{w}\left|Q_{k}\right| \vec{v}\right\rangle$ vanish for most cases, only a few terms of the $U$-matrix survive since $\langle\vec{u} \mid \vec{v}\rangle=\delta_{\vec{u}, \vec{v}}$ and $\left\langle\vec{w}\left|Q_{K}\right| \vec{v}\right\rangle=0$, unless $w_{K}=v_{K}+1$ or $v_{K}-1$ and $w_{L}=v_{L} \forall L \neq K$. In the case of $w_{K}=v_{K}+1$,

$$
\left\langle\vec{w}\left|Q_{K}\right| \vec{v}\right\rangle=\left[\frac{\left(v_{K}+1\right) \hbar}{2 \mu_{K} \Omega_{K}}\right]^{1 / 2},
$$

and for $w_{K}=v_{K}-1$,

$$
\left\langle\vec{w}\left|Q_{K}\right| \vec{v}\right\rangle=\left[\frac{v_{K} \hbar}{2 \mu_{K} \Omega_{K}}\right]^{1 / 2} .
$$

Thus, the matrix elements of the time evolution operator vanish for most final vibrational states.

The $0^{\text {th }}$ order vibronic state energies

To completely evaluate the first order vibronic states (27), we also need the $0^{\text {th }}$ order energies of the molecular states $E_{l_{v} \vec{v}}$, which are the sum of electronic energies $E_{l_{v}}^{0}$ plus the 
vibrational energies, $E_{\bar{v}}$, given by

$$
E_{\vec{v}}=\sum_{K=1}^{3 P-6}\left(v_{K}+\frac{1}{2}\right) \hbar \Omega_{K}
$$

Thus, the $0^{\text {th }}$ order molecular state energies are

$$
E_{l_{v} \vec{v}}=E_{l_{v}}^{0}+\sum_{K=1}^{3 P-6}\left(v_{K}+\frac{1}{2}\right) \hbar \Omega_{K}
$$

We may now use the $1^{\text {st }}$ order vibronic molecular states and energies to evaluate the expressions for different types of molecular electromagnetic spectra.

\section{Absorption spectra with vibrational structure}

The probability amplitude for light absorption is given by

$$
U_{f, n_{k}^{\lambda}-1 ; i, n_{k}^{\lambda}}=-i\left[2 \pi \hbar \omega_{k} n_{k}^{\lambda}\right]^{1 / 2} e^{i \vec{k} \cdot \vec{r}_{0}} \frac{\left\langle f\left|\hat{e}^{\lambda} \cdot \vec{\mu}\right| i\right\rangle}{\varepsilon_{f}-\varepsilon_{i}-\hbar \omega_{k}}\left(1-e^{i\left(\varepsilon_{f}-\varepsilon_{i}-\hbar \omega\right)\left(t-t_{0}\right) / \hbar}\right),
$$

where the states $|f\rangle$ and $|i\rangle$ are the exact molecular energy eigenstates. However, instead of the exact molecular eigenstates, we will use our first-order approximate vibronic states given in Eq (27). These vibronic states are better molecular states than the crude Born-Oppenheimer product states, $\left|l_{v}\right\rangle|\vec{u}\rangle$, which were our $0^{\text {th }}$ order molecular stationary states.

We will assume the initial molecular state $|i\rangle$ is a ground state vibrational level with vibronic wavefunction, 


$$
\begin{aligned}
|i\rangle=|g \vec{v}\rangle=|g\rangle|\vec{v}\rangle & -\sum_{j, \alpha} \sum_{\vec{w}} \sum_{K} \frac{\left\langle j_{\alpha}\left|\left(\frac{\partial H}{\partial Q_{K}}\right)_{0}\right| g\right\rangle\left\langle\vec{w}\left|Q_{K}\right| \vec{v}\right\rangle}{E_{g \vec{v}}-E_{j_{\alpha} \vec{w}}}\left|j_{\alpha}\right\rangle|\vec{w}\rangle f_{j_{\alpha} \vec{w}, g \vec{v}}\left(t_{0}-t_{0}^{\prime}\right) \\
& -\sum_{j, \alpha} \sum_{\vec{w}} \sum_{K, L} \frac{\left\langle j_{\alpha}\left|\left(\frac{\partial^{2} H^{\prime}}{\partial Q_{K} \partial Q_{L}}\right)_{0}\right| g\right\rangle\left\langle\vec{w}\left|Q_{K} Q_{L}\right| \vec{v}\right\rangle}{E_{g \vec{v}}-E_{j_{\alpha} \vec{w}}}\left|j_{\alpha}\right\rangle|\vec{w}\rangle f_{j_{\alpha} \vec{w}, g \vec{v}}\left(t_{0}\right) .
\end{aligned}
$$

We also need the arbitrary final state $\langle f|=\left\langle l_{v} \vec{u}\right|$, which is the adjoint of $\left|l_{v} \vec{u}\right\rangle$,

$$
\begin{aligned}
&\langle f|=\left\langle l_{v} \vec{u}\right|=\left\langle l_{v}\right|\langle\vec{u}|-\sum_{j, \alpha} \sum_{\bar{w}} \sum_{K} \frac{\left\langle l_{v}\left|\left(\frac{\partial H^{\prime}}{\partial Q_{K}}\right)_{0}\right| j_{\alpha}\right\rangle\left\langle\vec{w}\left|Q_{K}\right| \vec{u}\right\rangle}{E_{l_{v} \vec{u}}-E_{j_{\alpha} \bar{w}}}\left\langle j_{\alpha}\right|\langle\vec{u}| f_{l_{v}, j_{\alpha} \vec{w}} \\
&-\sum_{j, \alpha} \sum_{\bar{w}} \sum_{K, L} \frac{\left\langle l_{v}\left|\left(\frac{\partial^{2} H^{\prime}}{\partial Q_{K} \partial Q_{L}}\right)_{0}\right| j_{\alpha}\right\rangle\left\langle\vec{w}\left|Q_{K} Q_{L}\right| \vec{u}\right\rangle}{E_{l_{\nu} \vec{u}}-E_{j_{\alpha} \vec{w}}}\left\langle j_{\alpha}\right|\langle\vec{w}| f_{l_{v} \vec{u}, j_{\alpha}, \bar{w}} .
\end{aligned}
$$

The final state $\left|l_{v} \vec{u}\right\rangle$ at time $t$ can be a different vibrational level of the electronic ground state (infrared, Raman) or an excited electronic and vibrational state (UV-visible absorption, NIR).

Substituting these expressions for the initial and final states $|g \vec{v}\rangle$ and $\left\langle l_{v} \vec{u}\right|$ into the expression (40) for the absorption probability amplitude, we have

$$
\begin{aligned}
& U_{l_{v}, \vec{u}, g \hat{v}}^{n_{k}^{\lambda}-1, n_{k}^{\lambda}}=-i\left[2 \pi \hbar \omega_{k} n_{k}^{\lambda}\right]^{1 / 2} e^{i \vec{k} \cdot \vec{r}_{0}}\left[\frac{\left\langle l_{v}\left|\hat{e}^{\lambda} \cdot \vec{\mu}\right| g\right\rangle\langle\vec{u} \mid \vec{v}\rangle}{E_{l_{v} \vec{u}}-E_{g \vec{v}}-\hbar \omega_{k}}\left(1-e^{i\left(E_{l_{v}, \vec{u}}-E_{g \vec{v}}-\hbar \omega_{k}\right)\left(t-t_{0}\right) / \hbar}\right)\right.
\end{aligned}
$$

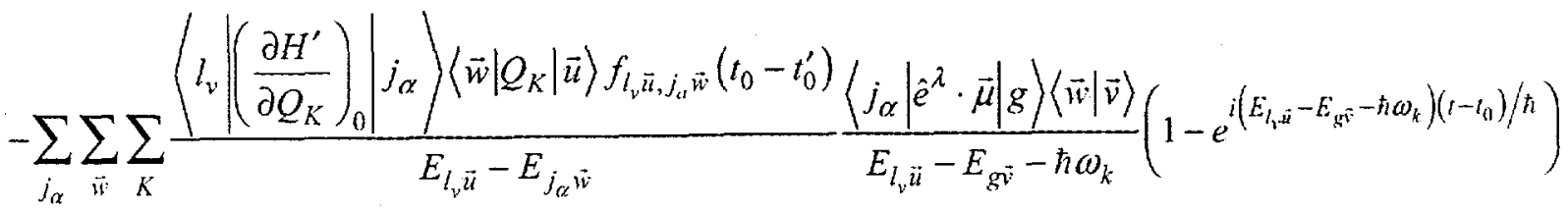




$$
\begin{aligned}
& -\sum_{j_{\alpha}} \sum_{\vec{w}} \sum_{K} \frac{\left\langle j_{\alpha}\left|\left(\frac{\partial H^{\prime}}{\partial Q_{K}}\right)_{0}\right| g\right\rangle\left\langle\bar{w}\left|Q_{K}\right| \vec{v}\right\rangle f_{j_{a} \vec{w}, g \vec{v}}\left(t_{0}-t_{0}^{\prime}\right)}{E_{g \vec{v}}-E_{j_{\alpha} \vec{w}}} \frac{\left\langle l_{v}\left|\hat{e}^{\lambda} \cdot \vec{\mu}\right| j_{\alpha}\right\rangle\langle\vec{u} \mid \vec{w}\rangle}{E_{l_{v} \vec{u}}-E_{g \vec{v}}-\hbar \omega_{k}}\left(1-e^{i\left(E_{l_{v}}-E_{g \bar{v}}-\hbar \omega_{k}\right)\left(t-t_{0}\right) / \hbar}\right) \\
& \left.+ \text { terms in } Q_{K} Q_{L} \cdots\right] \text {. }
\end{aligned}
$$

We neglect the fourth term with factors like $\left\langle\bar{w}\left|Q_{K}\right| \vec{v}\right\rangle\left\langle\vec{u}\left|Q_{K}\right| \vec{x}\right\rangle$ and other terms in $Q_{K} Q_{L}$.

Here, we have approximated $\left\langle\vec{u}\left|\left\langle l_{v}\left|\hat{e}^{\lambda} \cdot \vec{\mu}\right| j_{\alpha}\right\rangle\right| \vec{w}\right\rangle$ by $\left\langle l_{v}\left|\hat{e}^{\lambda} \cdot \vec{\mu}\right| j_{\alpha}\right\rangle\langle\vec{u} \mid \vec{w}\rangle$. The dipole operator is defined by $\vec{\mu} \equiv \sum_{\alpha} Z_{\alpha} e \vec{r}_{\alpha}$, where $\alpha$ includes all charged particles of the molecule (electrons and nuclei), thus, we have ignored the nuclear coordinate dependence of $\vec{\mu}$. In this case, nuclear coordinate dependence will come entirely through the $Q_{K}$-dependence of the energy eigenstates. This is reasonable for optical frequencies of light since the field primarily interacts with the light electrons, but not the heavy and shielded nuclei. On the other hand for IR absorption, this approximation is not sufficient and we must keep the explicit nuclear coordinate dependence of $\vec{\mu}$.

Each state absorbs independently of the other states, thus the probability of absorption to all of the molecular states in terms of the $U$-matrix is

$$
P\left(\hbar \omega_{k}\right)=\sum_{l_{v}, \vec{v}}\left|U_{l_{v} \vec{u}, g \bar{v}}^{n_{k}^{\lambda}-1, \bar{k}_{k}^{\lambda}}\right|^{2} .
$$

Evaluating the vibrational matrix elements and molecular state energies in $\mathrm{Eq}(43)$, we obtain non-vanishing terms for only three final vibrational states. 
(1) First, consider the case when $\vec{u}=\vec{v}$ and $l$ is an electronic excited state; this is called the purely electronic or 0-0 transition. In this case, we have

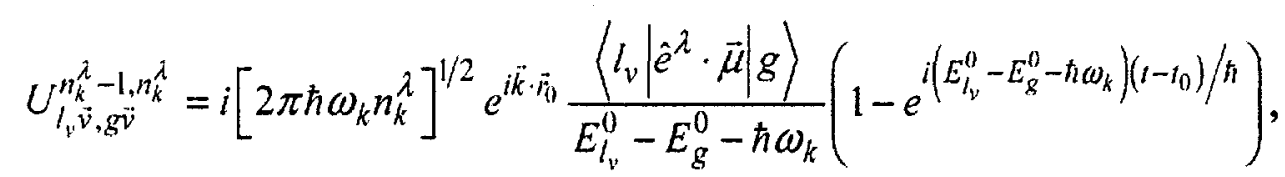

where the second and third terms vanish for $\vec{u}=\vec{v}$ because

$$
\left\langle\vec{w}\left|Q_{K}\right| \vec{v}\right\rangle\langle\vec{w} \mid \vec{v}\rangle=\left\langle\vec{w}\left|Q_{K}\right| \vec{v}\right\rangle \delta_{\vec{w}, \vec{v}}=\left\langle\vec{v}\left|Q_{K}\right| \vec{v}\right\rangle \delta_{\vec{w}, \vec{v}}=0
$$

(2) Next, consider the case, $|\vec{u}\rangle=\left|v_{1}, \cdots, v_{L-1}, v_{L}+1, \cdots, v_{3 N-6}\right| \equiv\left|v_{L}+1\right\rangle$, for which we get

$$
\begin{aligned}
& U_{l_{v}, v_{L}+1, g v_{L}}^{n_{i}^{i}-1 . n_{k}^{i}}=i\left[\frac{2 \pi n_{k}^{\lambda} \hbar \omega_{k}\left(v_{L}+1\right) \hbar}{2 \mu_{L} \Omega_{L}}\right]^{1 / 2} e^{i \vec{k} \cdot \hat{r}_{0}} \frac{\left(1-e^{i\left(E_{l_{v}}^{0}-E_{g}^{0}+\hbar \Omega_{L}-\hbar \omega_{k}\right)\left(t-t_{0}\right) / \hbar}\right)}{\left(E_{l_{v}}^{0}-E_{g}^{0}+\hbar \Omega_{L}-\hbar \omega_{k}\right)} \\
& \times \sum_{j_{\alpha}}\left[\frac{\left\langle l_{v}\left|\left(\frac{\partial H^{\prime}}{\partial Q_{L}}\right)_{0}\right| j_{\alpha}\right\rangle\left\langle j_{\alpha}\left|\hat{e}^{\lambda} \cdot \vec{\mu}\right| g\right\rangle f_{l_{\nu} v_{L}+1, j_{\alpha} v_{L}}}{E_{l_{v}}^{0}-E_{j_{k}}^{0}+\hbar \Omega_{L}}+\frac{\left\langle j_{\alpha}\left|\left(\frac{\partial H^{\prime}}{\partial Q_{L}}\right)_{0}\right| g\right\rangle\left\langle l_{v}\left|\hat{e}^{\lambda} \cdot \vec{\mu}\right| j_{\alpha}\right\rangle f_{j_{\alpha} v_{L}+1, g v_{L}}}{E_{g}^{0}-E_{j_{\alpha}}^{0}-\hbar \Omega_{L}}\right] .
\end{aligned}
$$

(3) The only other case in which an element of $U$ survives is when the final vibrational state is $|\vec{u}\rangle=\left|v_{1}, \cdots, v_{L-1}, v_{L}-1, \cdots, v_{3 N-6}\right|=\left|v_{L}-1\right\rangle$. In this case, we have

$$
\begin{aligned}
& U_{l_{v} \nu_{L}-1, n_{k} n_{L}}^{n_{k}-1, n_{L}^{i}}=i\left[\frac{2 \pi n_{k}^{\lambda} \hbar \omega_{k} v_{L} \hbar}{2 \mu_{L} \Omega_{L}}\right]^{1 / 2} e^{i \vec{k} \cdot \vec{r}_{0}} \frac{\left(1-e^{i\left(E_{l_{v}}^{0}-E_{k}^{0}-\hbar \Omega_{L}-\hbar \hbar \omega_{k}\right)\left(t-t_{0}\right) / \hbar}\right)}{\left(E_{l_{v}}^{0}-E_{g}^{0}-\hbar \Omega_{L}-\hbar \omega_{k}\right)} \\
& \times \sum_{j_{n}}\left[\frac{\left\langle j_{\alpha}\left|\left(\frac{\partial H^{\prime}}{\partial Q_{L}}\right)_{0}\right| l_{v}\right\rangle\left\langle j_{\alpha}\left|\hat{e}^{\hat{\lambda}} \cdot \vec{\mu}\right| g\right\rangle f_{l_{v} v_{L}-1, j_{\alpha} v_{L}}}{E_{l_{v}}^{0}-E_{j_{\alpha}}^{0}-\hbar \Omega_{L}}+\frac{\left\langle j_{\alpha}\left|\left(\frac{\partial H^{\prime}}{\partial Q_{L}}\right)_{0}\right| g\right\rangle\left\langle l_{v}\left|\hat{e}^{\lambda} \cdot \vec{\mu}\right| j_{\alpha}\right\rangle f_{j_{\alpha} v_{L}-1, g_{v_{L}}}}{E_{g}^{0}-E_{j_{\alpha}}^{0}+\hbar \Omega_{L}}\right] .
\end{aligned}
$$




\section{The zero-temperature approximation}

Before we examine what these three expressions say about the vibrational structure in electronic absorption spectra, let's make one additional simplification. At absolute zero temperature, only the lowest lying vibrational state will be populated, i.e., $|\vec{v}\rangle=|\overrightarrow{0}\rangle$, and under these conditions the three expressions become

$$
\begin{aligned}
& U_{l_{v}, n_{k} \overline{0}}^{n_{k}^{\lambda}-1, n_{k}^{\lambda}}=i\left[2 \pi n_{k}^{\lambda} \hbar \omega_{k}\right]^{1 / 2} e^{i \vec{k} \cdot \overline{r_{0}}}\left\langle l_{v}\left|\hat{e}^{\lambda} \cdot \vec{\mu}\right| g\right\rangle \frac{\left(1-e^{\left(E_{l_{v}}^{0}-E_{g}^{0}-\hbar \omega_{k}\right)\left(t-t_{0}\right) / \hbar}\right)}{\left(E_{l_{v}}^{0}-E_{g}^{0}-\hbar \omega_{k}\right)} \\
& U_{l_{v} l_{L}, g \theta_{l}}^{n_{i}^{\lambda}-1, n_{k}^{\lambda}}=i\left[\frac{\pi n_{k}^{\lambda} \hbar \omega_{k} \hbar}{\mu_{L} \Omega_{L}}\right]^{1 / 2} e^{i k \cdot \bar{n}_{0}} \frac{\left(1-e^{i\left(E_{l_{v}}^{0}-E_{g}^{0}+\hbar \Omega_{L}-\hbar \omega_{k}\right)\left(t-t_{0}\right) / \hbar}\right)}{\left(E_{l_{v}}^{0}-E_{g}^{0}+\hbar \Omega_{L}-\hbar \omega_{k}\right)} \\
& \times \sum_{j_{\alpha}}\left[\frac{\left\langle j_{\alpha}\left|\left(\frac{\partial H^{\prime}}{\partial Q_{L}}\right)_{0}\right| l_{v}\right\rangle\left\langle j_{\alpha}\left|\hat{e}^{\lambda} \cdot \vec{\mu}\right| g\right\rangle f_{l_{v} l_{L}, j_{\alpha} 0}}{E_{l_{v}}^{0}-E_{j_{\alpha}}^{0}+\hbar \Omega_{L}}+\frac{\left\langle j_{\alpha}\left|\left(\frac{\partial H^{\prime}}{\partial Q_{L}}\right)_{0}\right| g\right\rangle\left\langle l_{v}\left|\hat{e}^{\lambda} \cdot \vec{\mu}\right| j_{\alpha}\right\rangle f_{j_{\alpha} l_{L}, g 0}}{E_{g}^{0}-E_{j_{\alpha}}^{0}-\hbar \Omega_{L}}\right]
\end{aligned}
$$

and for the third case

$$
U_{l_{v}\left(-l_{L}\right), g 0}^{n_{k}^{\lambda}-1, n_{k}^{\lambda}}=0
$$

\section{Locations of the absorption band maxima}

We now use these three expressions to evaluate vibronic effects on optical absorption spectra. First, we will look at the $0^{\circ} \mathrm{K}$ approximation. Then we evaluate what the expression for case (3), which vanishes in the $0^{\circ} \mathrm{K}$ approximation, says about absorption spectra in cases where the temperature is not zero. 
When absorption is to an excited electronic state $\left|l_{v}\right\rangle$, one absorption band is given by Eq (49). $U_{l_{v}, g \overline{0}}^{n_{k}^{\lambda}-1, n_{k}^{\lambda}}$ peaks at $E_{l_{v}}^{0}-E_{g}^{0}=\hbar \omega_{k}$, that is, when the frequency of the incident light matches the purely electronic transition energy. (The intensity does not diverge at the resonance because of the imaginary line width term that should also appear in the denominator in Eq (49). The intensity at this peak is determined by the transition dipole matrix element, $\left\langle l_{v}\left|\hat{e}^{\lambda} \cdot \vec{\mu}\right| g\right\rangle$, the incident light intensity $I_{0}=n_{k}^{\lambda} \hbar \omega_{k}$, and the other factors in Eq (49). For an $A_{l g}$ ground state $|g\rangle$, it is sufficient for $\left|l_{v}\right\rangle$ to transform as $x, y$, or $z$ so that the dipole matrix element does not necessarily vanish. In the $D_{4 h}$ molecular symmetry of porphyrin for example, that would be either an $E_{u}$ or an $A_{2 u}$ state. We get a 0-0 absorption band for each electronic state with nonvarishing dipole matrix element.

Additional vibronic peaks appear in the absorption spectrum as determined by $U_{l_{v} 1_{L}, g_{0}}^{n_{k}^{\lambda}-1, n_{k}^{\lambda}}$ given by $\mathrm{Eq}(50)$. The peaks occur when the frequency of the incident light is $\hbar \omega_{k}=E_{l_{v}}^{0}-E_{g}^{0}+\hbar \Omega_{L}$. This is when the light energy corresponds to the energy separation of the ground and excited electronic levels plus the energy of the vibrational quantum for the $L^{\text {th }}$ normal mode. These vibrational side bands thus occur on the high-energy side of the $0-0$ band, and the intensity at resonance depends on the dipole matrix element, the same as the $0-0$ absorption band. However, the intensity of this so-called 0-1 absorption band also depends on the magnitude of the vibronic coupling matrix elements, $\left\langle j_{\alpha}\left|\left(\frac{\partial H^{\prime}}{\partial Q_{L}}\right)_{0}\right| l_{v}\right\rangle$. 
Absorption processes are independent events so the spectrum is the sum of all such transitions, i.e.,

$$
P\left(\hbar \omega_{k}\right)=\sum_{l_{v}} \sum_{\bar{u}}\left|U_{l_{v} \bar{u}, g \bar{v}}^{n_{k}^{\lambda}-1, n_{k}^{\lambda}}\right|^{2}=\sum_{l_{v}} \sum_{K}\left|U_{l_{v} 1_{L}, n^{2} \overline{0}}^{n_{1}^{\lambda}-1, n_{k}^{\lambda}}\right|^{2} .
$$

(See equations (11) and (12) in a paper by Shelnutt.)\{Shelnutt, 1981\#16132\} Vibrational side bands are illustrated in Figure 2.

Thus, the absorption spectrum, which is proportional to the probability of absorption, is composed of the 0-0 transition for each electronic state and a vibrational satellite band composed of the $0-1$ absorptions from each of the allowed normal coordinates, that is, normal coordinates for which the vibronic coupling matrix elements do not vanish. The normal coordinates that contribute to the spectrum are determined by the symmetry properties of the vibronic coupling matrix elements.

\section{Intensity borrowing in absorption spectra}

Ignoring the $t$-dependent factors, the intensity of the $0-0$ absorption band is

$$
I_{l_{v}, 0, g 0}=\frac{2 \pi \hbar \omega_{k} n_{k}^{\lambda}\left|\left\langle l_{v}\left|\hat{e}^{\lambda} \cdot \vec{\mu}\right| g\right\rangle\right|^{2}}{\left(E_{l_{v}}^{0}-E_{g}^{0}-\hbar \omega_{k}\right)^{2}},
$$

and the intensity of the contribution of $L^{\text {th }}$ normal mode to the vibrational side band is 


$$
\begin{aligned}
I_{l_{\nu} I_{L}, g 0} & =\frac{\pi n_{k}^{\lambda} \hbar \omega_{k}}{\mu_{L} \Omega_{L}} \frac{1}{\left(E_{l_{v}}^{0}-E_{g}^{0}+\hbar \Omega_{L}-\hbar \omega_{k}\right)^{2}} \\
& \times \mid \sum_{j_{\alpha}}\left[\frac{\left\langle j_{\alpha}\left(\frac{\partial H^{\prime}}{\partial Q_{L}}\right)_{0} \mid l_{v}\right\rangle\left\langle j_{\alpha}\left|\hat{e}^{\lambda} \cdot \vec{\mu}\right| g\right\rangle f_{l_{v} I_{L}, j_{\alpha} 0}}{E_{l_{v}}^{0}-E_{j_{\alpha}}^{0}+\hbar \Omega_{L}}+\left.\frac{\left\langle j_{\alpha}\left(\frac{\partial H^{\prime}}{\partial Q_{L}}\right)_{0} \mid g\right\rangle\left\langle l_{v}\left|\hat{e}^{\lambda} \cdot \vec{\mu}\right| j_{\alpha}\right\rangle f_{j_{\alpha} I_{L}, g 0}}{E_{g}^{0}-E_{j_{\alpha}}^{0}-\hbar \Omega_{L}}\right|^{2}\right.
\end{aligned}
$$

For electronic absorption by $\mathrm{D}_{4 \mathrm{~h}}$ metalloporphyrins, the energy denominator of the second term in the numerator is large, since

$$
E_{j_{\alpha}}^{0}-E_{g}^{0}+\hbar \Omega_{L}>E_{j_{\alpha}}^{0}-E_{g}^{0} \gg \hbar \Omega_{L}
$$

for $j_{\alpha} \neq g$. In the case $j_{\alpha}=g$, the matrix elements $\left\langle g\left|\left(\frac{\partial H^{\prime}}{\partial Q_{L}}\right)_{0}\right| g\right\rangle$ all vanish since the ground-state matrix elements are all zero. Thus, if we neglect the vibronic coupling to the ground state, then we have only the first term that survives with $j_{\alpha} \neq g$,

$$
I_{l_{\nu} 1_{L}, g 0}=\frac{2 \pi n_{k}^{\lambda} \hbar \omega_{k} \hbar}{\mu_{L} \Omega_{L}}\left|\sum_{j_{\alpha}} \frac{\left\langle j_{\alpha}\left|\left(\frac{\partial H^{\prime}}{\partial Q_{L}}\right)_{0}\right| l_{\nu}\right\rangle\left\langle j_{\alpha}\left|\hat{e}^{\lambda} \cdot \vec{\mu}\right| g\right\rangle}{E_{l_{\nu}}^{0}-E_{j_{\alpha}}^{0}+\hbar \Omega_{L}}\right| \frac{1}{\left(E_{l_{\nu}}^{0}-E_{g}^{0}+\hbar \Omega_{L}-\hbar \omega_{k}\right)^{2}}
$$

Usually, the largest term in the sum of Eq (56) will be from vibronic coupling within an excited electronic state $\left|l_{v}\right\rangle$, i.e., the term for which $j_{\alpha}=l_{v}$, since this allows the denominator to get as small as $h \Omega_{L}$. However, nearby electronic states for which $E_{j_{\alpha}}^{0}-E_{l_{\nu}}^{0} \approx \hbar \Omega_{L}$ can also give a very large contribution. 
Even if an electronic state $\left|j_{\alpha}\right\rangle$ is farther away in energy (i.e., $E_{j_{\alpha}}^{0}-E_{l_{\nu}}^{0}>\hbar \Omega_{L}$ ), vibronic coupling to $\left|j_{\alpha}\right\rangle$ will contribute if its transition dipole $\left\langle j_{\alpha}\left|\hat{e}^{\lambda} \cdot \vec{\mu}\right| g\right\rangle$ is large enough to make up for the large denominator. When this occurs, it is called intensity borrowing and intensity borrowing plays an important role in the absorption spectra of porphyrins.

Interference effects on the relative intensities of the $0-0$ and $0-1$ bands

Another feature of the absorption spectra of porphyrins is that the intensity factor of Eq (56) may contain more than one term. In this case, cancellation or addition of the contributions to the vibrational side band from both the intra-state and inter-state terms may occur. Interference is important in determining a particular vibration's contribution to the $0-1$ absorption band. These effects are most noticeable when the magnitudes of the terms are nearly equal. Their influence is even more apparent for the resonance Raman excitation profiles than for the absorption spectra.

\section{Optical absorption spectra of metalloporphyrins}

The UV-visible absorption spectra of metalloporphyrins, illustrated in Figure 3 provide a good example of intensity borrowing. Part of the intensity in $Q_{v}$-band is due to vibronic coupling among the $Q_{x}$ and $Q_{y}$ components of the doubly degenerate $E_{u}$ state $\left(\mathrm{D}_{4 \mathrm{~h}}\right.$ molecular symmetry), i.e.,

$$
j_{\alpha}=l_{\nu}=Q_{x}, Q_{y} \quad(j=\mathrm{Q} ; \alpha=x, y)
$$

through matrix elements such as 


$$
\left\langle Q_{x}\left|\left(\frac{\partial H^{\prime}}{\partial Q_{L}}\right)_{0}\right| Q_{x}\right\rangle
$$

Another part of the intensity is a result of coupling of the $Q$ states to the $B_{x}$ and $B_{y}$ states, i.e., $j_{\alpha}=B_{x}, B_{y}$ and $l_{v}=Q_{x}, Q_{y}$ by matrix elements like

$$
\left\langle B_{y}\left|\left(\frac{\partial H^{\prime}}{\partial Q_{L}}\right)_{0}\right| Q_{x}\right\rangle
$$

Even though the separation between the $Q$ and $B$ states is large $\left(\sim 7000 \mathrm{~cm}^{-1}\right)$ compared to typical fundamental vibrational frequencies $\left(\sim 1500 \mathrm{~cm}^{-1}\right)$, the large extinction coefficient of $B$ still allows it to contribute sometimes on par with the inter-Q state coupling.

\section{Symmetry considerations in the absence of ground-state distortion of the porphyrin}

For a $\mathrm{D}_{4 \mathrm{~h}}$-symmetric porphyrin, the totally symmetric representation must belong to $\Gamma_{l_{v}} \times \Gamma_{j_{\alpha}} \times \Gamma_{L}$ if the vibronic coupling matrix elements of the type (58) and (59) are to be nonzero. Since the $Q$ and $B$ states are of (transform according to) $\mathrm{E}_{\mathrm{u}}$ symmetry in $\mathrm{D}_{4 \mathrm{~h}}$, we can see from the character table (Table 1) that $E_{u} \otimes E_{u}$ contains $A_{1 g}, A_{2 g}, B_{1 g}$, and $B_{2 g}$. For the $x$ - and $y$-components of the same electronic state, the $\mathrm{A}_{2 \mathrm{~g}}$ symmetry representation must be omitted from these representations of the group since $\mathrm{A}_{2 \mathrm{~g}}$ does not transform like $x^{2}, y^{2}$, or $x y$ (see Table 1). Therefore, for the vibronic coupling matrix element to survive, $\Gamma_{L}$ crossed with one of these four (or three) representations must give the totally symmetric representation. This occurs only when the symmetry of the $L^{\text {th }}$ normal mode is itself of one of these symmetries because the totally symmetric representation is obtained when one of these symmetry representations is crossed with itself. Thus, only the $A_{1 g}, A_{2 g}, B_{1 g}$, and $B_{2 g}$ vibrational modes contribute to the 
absorbance of the vibration side band. Vibrations with all four of these symmetries contribute for coupling between the $Q$ and $B$ states; only $\mathrm{A}_{1 \mathrm{~g}}, \mathrm{~B}_{1 \mathrm{~g}}$, and $\mathrm{B}_{2 \mathrm{~g}}$ vibrational modes contribute by vibronically coupling within the $\left(Q_{\mathrm{x}}, Q_{\mathrm{y}}\right)$ and $\left(B_{\mathrm{x}}, B_{\mathrm{y}}\right)$ pairs. Since the same vibronic and electronic matrix elements also determine the symmetries of the vibrations that are active in the resonance Raman spectra, the same selection rules determine the Raman active modes.

\section{Connection with the ground-state deformations of porphyrin from NSD}

The displacements along the normal coordinates for the ground state structures of porphyrins can be explicitly represented in the above-described development of the vibronic states of molecules. Remember that these displacements are measured from a $\mathrm{D}_{4 \mathrm{~h}}$-symmetric porphyrin reference structure, and the displacements are for the ground state of the porphyrin. This situation is treated by carrying out the Taylor series expansion about the reference structure instead of the equilibrium structure of the molecule. In this case, the linear matrix elements of the type $\left\langle g\left|\left(\frac{\partial H}{\partial Q_{L}}\right)_{0}\right| g\right\rangle$ no longer vanish. We will also make the approximation that keeping one or several of these linear matrix elements adequately describes the distortion in the ground state. This means that the development of the vibronic theory up to this point is valid as long as we now include these ground-state vibronic matrix elements.

The connection with NSD is made if the reference structure for the expansion is the same as the NSD reference structure. Before we continue we must first understand the meaning of the normal coordinates in this instance. The NSD displacements are along the coordinates of the "bare" metalloporphyrin macrocycle, i.e., the copper porphyrin macrocycle for which the masses

of the 12 hydrogen atom substituents and the metal atom are set to zero. For all substituted 
porphyrins these bare macrocycle normal coordinates are mixed with the substituent and metal motions to give the true normal coordinates of the particular substituted porphyrin. Thus, our expressions in terms of the macrocycle modes describe how a normal mode of the substituted porphyrin responds to a macrocycle distortion to the extent that it contains contributions from the normal coordinates of the bare macrocycle. We will often find that the ground state distortion occurs along only one or two macrocycle normal coordinates. The analysis that we now describe strictly only applies for a distortion of our bare reference macrocycle, which has no physical reality; however, mixing of the macrocycle and substituent (and metal) motions insures that the actual porphyrin normal modes behave similarly. If the normal coordinate vectors are know for the porphyrin, then we can used NSD to project out the contributions of the bare macrocycle modes.

We must remember that the Hamiltonian itself is no longer totally symmetric because of the inclusion of the symmetry-lowering terms. For the ground-state vibrational states, the Harniltonian is now given by

$$
H^{e}=T_{N}+E_{g}^{0}+\sum_{L} Q_{L}\left\langle g\left|\left(\frac{\partial H^{e}}{\partial Q_{L}}\right)_{0}\right| g\right\rangle+1 / 2 \sum_{L} Q_{L}^{2} f_{L}
$$

instead of the Hamiltonian in Eq (17), which applies when the expansion is about the equilibrium undistorted structure. The state $|g\rangle$ is the electronic ground state of the $\mathrm{D}_{4 \mathrm{~h}}$ reference structure in the NSD analysis and is assumed to be $A_{1 g}$. At first it might seem that the matrix elements $\left\langle g\left|\left(\frac{\partial H}{\partial Q_{L}}\right)_{0}\right| g\right\rangle$ actually vanish since the $0^{\text {th }}$ order electronic state $|g\rangle$ is $A_{1 g}$ in $D_{4 h}$, thus the matrix element would vanish unless $\left(\frac{\partial H}{\partial Q_{L}}\right)_{0}$ is also $A_{1 g}$. This seems to allow only totally 
symmetric deformations and no symmetry lowering. However, remember that $H^{e}$ is no longer totally symmetric when there is a deformation along a particular asymmetric coordinate $Q_{L}$. This means that $\left(\frac{\partial H}{\partial Q_{L}}\right)_{0}$ contains $A_{1 g}$ as long as $\Gamma_{L}$ is contained in the representations of the Hamiltonian. If the distortion is along only one normal coordinate, say $Q_{K}$, then the Hamiltonian contains $\mathrm{A}_{1 \mathrm{~g}} \oplus \Gamma_{K}$, and thus $\Gamma_{L} \otimes \Gamma_{H^{e}}=\Gamma_{L} \otimes\left(\mathrm{A}_{1 \mathrm{~g}} \oplus \Gamma_{K}\right)$ will contain $\mathrm{A}_{1 \mathrm{~g}}$ if $\Gamma_{L}$ is either $\mathrm{A}_{\mathrm{lg}}$ or $\Gamma_{K}$. That is, the matrix element will not vanish for normal modes of the same symmetry as the normal coordinate along which the ground state distortion occurs.

The molecular symmetry of the porphyrin is lowered by the distortion, and if the distortion is along a single non-totally symmetric normal coordinate $Q_{K}$, then the molecular symmetry is that of the highest symmetry point group for which $\Gamma_{K}$ becomes totally symmetric. This point group can be determined using the correlation table for the $\mathrm{D}_{4 \mathrm{~h}}$ point group and its subgroups (Table 2). For example, consider a purely ruffled porphyrin that is distorted along the lowest-frequency normal coordinate of $B_{1 u}$ symmetry. Examination of the correlation table shown in Table 2 indicates that the point group $\mathrm{D}_{2 \mathrm{~d}}\left(C_{2}^{\prime}\right)$ has $\mathrm{B}_{1 \mathrm{u}}$ alone of all the non-totally symmetric modes going into $A_{1}$. Thus, pure ruffling gives $D_{2 d}$ for the macrocycle symmetry. If the ground-state distortion is along more than one normal coordinate, then the distortion is lowered to the point group for which the symmetries of all of these normal modes become totally symmetric. For example, the gabled porphyrin structure involves deformations along both the ruffling and doming coordinates with symmetries $B_{1 u}$ and $A_{2 u}$, respectively. From the correlation table, the point group $\mathrm{C}_{2 \mathrm{v}}\left(C_{2}, \sigma_{d}\right)$ has both $\mathrm{B}_{1 \mathrm{u}}$ and $\mathrm{A}_{2 \mathrm{u}}$ as $\mathrm{A}_{1}$. Thus, the symmetry of the macrocycle and of course its molecular Hamiltonian are $\mathrm{C}_{2 \mathrm{v}}$. 
The equations we have derived for UV-visible absorption spectra by expanding about the equilibrium structure of the molecule are almost the same as those needed for an expansion about the $D_{4 h}$ reference structure. However, there are more nonvanishing matrix elements to be concerned with, mainly the linear terms of the ground electronic state. These matrix elements are directly related to the deformation along the normal coordinates. From Eq (17), the minimum of the potential energy (where the slope is zero) is at $Q_{L}=0$ for all $L$. In contrast, the minimum in the potential energy for $\mathrm{Eq}(60)$ occurs where the condition

$$
\frac{\partial H^{e}}{\partial Q_{K}}=\left\langle g\left|\left(\frac{\partial H^{e}}{\partial Q_{K}}\right)_{0}\right| g\right\rangle+Q_{K}^{0} f_{K}=0
$$

is met. Thus, the deformation along the $K^{\text {th }}$ normal coordinate $Q_{K}^{0}$ is

$$
Q_{K}^{0}=\frac{-\left\langle g\left|\left(\frac{\partial H^{e}}{\partial Q_{K}}\right)_{0}\right| g\right\rangle}{f_{K}}
$$

The energy of the equilibrium point of the potential energy is lower than $E_{g}^{0}$ by

$$
\frac{\left|\left\langle g\left|\left(\frac{\partial H^{e}}{\partial Q_{K}}\right)_{0}\right| g\right\rangle\right|^{2}}{8 f_{K}} .
$$

The deformations $Q_{K}^{0}$ are determined in the NSD analysis. However, keep in mind that these are the displacements along the normal coordinates of the reference macrocycle (no substituents or metal), not the normal coordinates of a particular substituted porphyrin. Strictly speaking, since no quadratic terms are added in Eq (60), then the normal coordinates of the bare macrocycle are unaltered by the distortion. 


\section{Effects of ground-state distortion on the UV-visible absorption spectra}

At first order, the $0-0$ transition is unaffected by the distortion in the ground state because the vibronic matrix elements do not appear in $\mathrm{Eq}(53)$. At higher orders of the expansion in $\mathrm{Eq}$ (43), new bilinear terms will introduce distortion effects on the 0-0 absorption bands.

For the $0-1$ transitions of excited electronic states $\left|l_{v}\right\rangle$ such as Q and B for porphyrins, the intensity factor for $\mathrm{Eq}(54)$ contains the term,

$$
\frac{\left\langle g\left|\left(\frac{\partial H}{\partial Q_{L}}\right)_{0}\right| l_{v}\right\rangle\left\langle g\left|\hat{e}^{\lambda} \cdot \vec{\mu}\right| g\right\rangle f_{l_{v} 1_{L}, g 0}}{E_{l}^{0}-E_{g}^{0}+\hbar \Omega_{L}}+\frac{\left\langle g\left|\left(\frac{\partial H}{\partial Q_{L}}\right)_{0}\right| g\right\rangle\left\langle l_{\nu}\left|\hat{e}^{\lambda} \cdot \vec{\mu}\right| j_{\alpha}\right\rangle f_{g 1_{L}, g 0}}{-\hbar \Omega_{L}}
$$

when $j_{\alpha}=g$ in the sum. Usually, the first term is small because the electronic states $l_{v}\left(\mathrm{Q}_{\mathrm{x}}, \mathrm{Q}_{\mathrm{y}}\right.$, $\mathrm{B}_{\mathrm{x}}, \mathrm{B}_{\mathrm{y}}$ ) are far above the ground state, making the denominator large. In addition, the second term usually vanishes in the absence of ground state distortion because the vibronic matrix elements $\left\langle g\left|\left(\frac{\partial H}{\partial Q_{K}}\right)_{0}\right| g\right\rangle$ vanish. However, when there is ground state distortion, the matrix elements $\left\langle g\left|\left(\frac{\partial H}{\partial Q_{K}}\right)_{0}\right| g\right\rangle$ do not vanish, and the modes along which the distortion occurs then contribute to the absorbance of the vibrational side band of the electronic transition by way of the last term in Eq (64). The new vibrations that are activated by this mechanism contribute peaks at the frequency of the 0-0 transition plus the vibrational frequency of the normal mode. For porphyrins, the symmetry of these new normal modes are not restricted to those that couple $\mathrm{E}_{u}$ electronic states, but are of the symmetry of the deformations contributing to the ground state distortion. For example, porphyrin doming is an $A_{2 u}$ deformation, thus $A_{2 u}$ normal coordinates containing the dom vibration can contribute to the vibrational satellites of the $\mathrm{Q}$ and $\mathrm{B}$ absorption 
bands of the domed porphyrin. The magnitude of the contribution depends on the magnitude of $\left\langle g\left|\left(\frac{\partial H}{\partial Q_{L}}\right)_{0}\right| g\right\rangle$ and thus the displacement along the $L^{\text {th }}$ normal coordinate, i.e., the dom deformation in this case. Physically, this just expresses the obvious result that the totally symmetric modes in the lowered symmetry of the distorted porphyrin are allowed.

Other than the addition of the $j_{\alpha}=g$ term of $\mathrm{Eq}(64)$, the expression for the $0-1$ term remains formally the same as given in Eq (56). However, the distortion introduces additional nonzero matrix elements in the sum that mix $\left|j_{\alpha}\right\rangle$ and $\left|l_{v}\right\rangle$ and thus alter the contributions of the normal modes to the absorption and resonance Raman spectra. In the case of $\mathrm{D}_{4 \mathrm{~h}}$-symmetric porphyrins, only in-plane vibrations of $A_{1 g}, A_{2 g}, B_{1 g}$, and $B_{2 g}$ influence the vibrational side bands, because these symmetries are contained in $\mathrm{E}_{\mathrm{u}} \otimes \mathrm{E}_{\mathrm{u}}$. That is, $\left(\frac{\partial H}{\partial Q_{L}}\right)_{0}$, which transforms like $\Gamma_{L}$, must have one of these symmetry classifications if the $L^{\text {th }}$ mode is to contribute by mixing two $E_{u}$ states like $Q$ and $B$.

On the other hand, if the molecule is distorted along say the $K$ th normal coordinate, then $H$ has the symmetry $A_{1 g} \otimes \Gamma_{K} . \quad$ Thus, $\left(\frac{\partial H}{\partial Q_{L}}\right)_{0}$ has the symmetry $\Gamma_{L} \otimes \Gamma_{H}=\Gamma_{L} \otimes\left(A_{1 \mathrm{~g}} \oplus \Gamma_{K}\right)=\Gamma_{L} \oplus\left(\Gamma_{L} \otimes \Gamma_{K}\right)$. The matrix elements coupling the $\mathrm{Q}$ and B $\mathrm{E}_{\mathrm{u}^{-}}$ symmetry transitions, $\left\langle l_{v}\left|\left(\frac{\partial H}{\partial Q_{L}}\right)_{0}\right| j_{\alpha}\right\rangle$, to be allowed also require that $\Gamma_{L} \oplus\left(\Gamma_{L} \otimes \Gamma_{K}\right)=\mathrm{E}_{u} \otimes \mathrm{E}_{\mathrm{u}}$ $=A_{1 g} \oplus A_{2 g} \oplus B_{1 g} \oplus B_{2 g}$. This condition is satisfied if

$$
\Gamma_{L}=A_{1 g}, A_{2 g}, B_{1 g}, \text { or } B_{2 g}
$$

or 


$$
\Gamma_{L} \otimes \Gamma_{K}=\mathrm{A}_{1 \mathrm{~g}}, \mathrm{~A}_{2 \mathrm{~g}}, \mathrm{~B}_{1 \mathrm{~g}}, \text { or } \mathrm{B}_{2 \mathrm{~g}}
$$

The former case is just the usual selection rule making the $A_{1 g}, A_{2 g}, B_{1 g}$, and $B_{2 g}$ modes active for the vibronic side bands of the $\mathrm{Q}$ and $\mathrm{B}$ transitions. In the latter case, $\mathrm{Eq}(66)$, the modes that can contribute depend on the symmetry of the deformation $\Gamma_{K}$. Since any of the representations $A_{1 g}, A_{2 g}, B_{1 g}$, and $B_{2 g}$ crossed with itself or one of the other members of this collection also gives one of this set, then any of these in-plane vibrations, which are already active in $\mathrm{D}_{4 \mathrm{~h}}$ symmetry, will have additional nonzero matrix elements brought about by the distortion. Furthermore, if the ground state deformation $\Gamma_{K}$ is of $E_{u}$ in-plane symmetry, then $E_{u}$ normal modes will contribute to the Raman and absorption spectra because Eq (66) is then satisfied. Similarly, if the distortion is an out-of-plane $\mathrm{E}_{\mathrm{g}}$ distortion (e.g., a wave deformation), the $\mathrm{E}_{\mathrm{g}}$ modes are activated. Lastly, for the other out-of-plane modes of $A_{1 u}, A_{2 u}, B_{1 u}$, and $B_{2 u}$ symmetries, we get one of $A_{1 g}, A_{2 g}, B_{1 g}$, and $B_{2 g}$ by crossing any one of the out-of-plane symmetries with one of the other members of this set. Thus, a distortion of one these types activates all of the other out-ofplane modes of this set of symmetries.

As a concrete example, doming is a deformation along the lowest frequency mode of $A_{2 u}$ symmetry, giving $\Gamma_{K}=A_{2 u}$. This activates all $A_{2 u}$ modes since $\Gamma_{L} \otimes \Gamma_{K}=A_{2 u} \otimes A_{2 u}=A_{1 g}$. However, $\mathrm{B}_{1 \mathrm{u}}$ modes are active as well since $\Gamma_{L} \otimes \Gamma_{K}=\mathrm{B}_{1 \mathrm{u}} \otimes \mathrm{A}_{2 \mathrm{u}}=\mathrm{B}_{2 \mathrm{~g}}$. Similarly, doming activates the other nondegenerate out-of-plane modes.

Summarizing, $A_{1 g}, A_{2 g}, B_{1 g}$, and $B_{2 g}$ modes are always active in vibronically coupling $Q$ and $B$ transitions of porphyrins, but deformation along one of these in-plane modes alters their contribution to the vibrational side bands of $Q$ and $B$. Normal modes of $A_{1 u}, A_{2 u}, B_{1 u}$, and $B_{2 u}$ 
symmetries will contribute if the ground state deformation is along a normal coordinate of one of these symmetry types. $E_{u}$ normal modes become active if the deformation is along a $E_{u}$ mode, and $\mathrm{E}_{\mathrm{g}}$ modes become active if the deformation is $\mathrm{E}_{\mathrm{g}}$. These contributions from matrix elements like $\left\langle l_{v}\left|\left(\frac{\partial H}{\partial Q_{L}}\right)_{0}\right| j_{\alpha}\right\rangle$ are independent of the contributions from the ground state matrix elements $\left\langle g\left|\left(\frac{\partial H}{\partial Q_{L}}\right)_{0}\right| g\right\rangle$ that will also be present as describe above. Notice that the former contributions

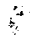
are not directly related to the latter ground-state distortion given by the NSD deformations. 


\section{ACKNOWLEDGEMENTS}

Sandia is a multiprogram laboratory operated by Sandia Corporation, a Lockheed Martin Company, for the United States Department of Energy under Contract DE-AC04-94AL85000. 


\section{REFERENCES AND FOOTNOTES}

1. Thus, the force given by

$$
F_{K}=-\frac{\partial E_{g}\left(Q_{K}\right)}{\partial Q_{K}}
$$

must vanish for all $K$ at the equilibrium point.

$$
\begin{gathered}
0=F_{L}=-\frac{\partial}{\partial Q_{L}}\left(E_{g}^{0}+\sum_{K} Q_{K}\left\langle g\left|\left(\frac{\partial H}{\partial Q_{K}}\right)_{0}\right| g\right\rangle+\frac{1}{2} \sum_{K} Q_{K}^{2}\left\langle g\left|\left(\frac{\partial^{2} H}{\partial Q_{K}^{2}}\right)_{0}\right| g\right\rangle\right) \\
=-\left\langle g\left|\left(\frac{\partial H}{\partial Q_{K}}\right)_{0}\right| g\right\rangle-Q_{L}\left\langle g\left|\left(\frac{\partial^{2} H}{\partial Q_{L}^{2}}\right)_{0}\right| g\right\rangle
\end{gathered}
$$

at $Q_{L}=0$, the force will vanish if and only if we have

$$
\left\langle g\left|\left(\frac{\partial H}{\partial Q_{L}}\right)_{0}\right| g\right\rangle=0, \quad \forall L .
$$

2. We must still show that a set of coordinates $Q_{K}$ can be found for which both the kinetic energy operator and the potential energy $E_{g}\left(Q_{K}\right)$ can be simultaneously written in the diagonal forms, i.e.,

$$
-\frac{\hbar^{2}}{2} \sum_{K} \frac{1}{\mu_{K}} \frac{\partial^{2}}{\partial Q_{K}^{2}}
$$

and

$$
\left.E_{g}^{0}+\frac{1}{2} \sum_{K} Q_{K}^{2} f_{K}\right)
$$

3. In the above we have used a result from perturbation theory. The state $\left|i\left(t_{0}\right)\right\rangle$ of the molecule at time $t_{0}$ after the vibrational-electronic interaction $H^{\prime}$ is turned on at $t_{0}^{\prime}$ is 
obtained from the coefficients

$$
\langle f \mid i(t)\rangle=\langle f|U| i\rangle=U_{f i} .
$$

This is the amplitude for the probability of starting in an initial $0^{\text {th }}$ order eigenstate $i$ and evolving to a $0^{\text {th }}$ order eigenstate $f$ in time $t_{0}^{\prime}$ to $t_{0}$, at which time the interaction with the field is turned on. The $0^{\text {th }}$ order state $i$ evolves according to

$$
|i(t)\rangle=\sum_{j}|j\rangle\langle j \mid i(t)\rangle=\sum_{j}|j\rangle\langle j|U| i\rangle=\sum_{j} U_{j i}|j\rangle .
$$

Now from our earlier result, to first order

$$
U_{j i}=\delta_{j i}+\frac{\left\langle j\left|H^{\prime}\right| i\right\rangle}{E_{j}-E_{i}}\left(1-e^{i\left(E_{j}-E_{i}\right)\left(t_{0}-t_{0}^{\prime}\right) / \hbar}\right)
$$

therefore, to first order the state at time $t_{0}$ is

$$
\begin{gathered}
\left|l\left(t_{0}\right)\right\rangle=\sum_{j} U_{j l}|j\rangle=\sum_{j}\left[\delta_{j l}+\left\langle j\left|H^{\prime}\right| l\right\rangle \frac{\left(1-e^{i\left(E_{j}-E_{l}\right)\left(t_{0}-t_{0}^{\prime}\right) / \hbar}\right)}{E_{j}-E_{l}}\right]|j\rangle \\
=|l\rangle+\sum_{j}\left\langle j\left|H^{\prime}\right| l\right\rangle \frac{\left(1-e^{i\left(E_{j}-E_{l}\right)\left(t_{0}-t_{0}^{\prime}\right) / \hbar}\right)_{E_{j}-E_{l}}|j\rangle .}{}
\end{gathered}
$$

Substituting for the vibronic Hamiltonian leads to the improved molecular eigenstates given above. 
Table 1. Character table for the $D_{4 h}$ molecular point group.

\begin{tabular}{l|cccccccccc|l|l}
$D_{4 h}$ & $\mathrm{E}$ & $2 C_{4}$ & $C_{2}$ & $2 C_{2}$ & $2 C_{2}$ & $i$ & $2 S_{4}$ & $\sigma_{h}$ & $2 \sigma_{v}$ & $2 \sigma_{d}$ & & \\
\hline$A_{1 g}$ & 1 & 1 & 1 & 1 & 1 & 1 & 1 & 1 & 1 & 1 & & $x^{2}+y^{2}, z^{2}$ \\
$A_{2 g}$ & 1 & 1 & 1 & -1 & -1 & 1 & 1 & 1 & -1 & -1 & & \\
$B_{1 g}$ & 1 & -1 & 1 & 1 & -1 & 1 & -1 & 1 & 1 & -1 & & $x^{2}-y^{2}$ \\
$B_{2 g}$ & 1 & -1 & 1 & -1 & 1 & 1 & -1 & 1 & -1 & 1 & & $x y$ \\
$E_{g}$ & 2 & 0 & -2 & 0 & 0 & 2 & 0 & -2 & 0 & 0 & & $(x z, y z)$ \\
$A_{1 u}$ & 1 & 1 & 1 & 1 & 1 & -1 & -1 & -1 & -1 & -1 & & \\
$A_{2 u}$ & 1 & 1 & 1 & -1 & -1 & -1 & -1 & -1 & 1 & 1 & $z$ & \\
$B_{1 u}$ & 1 & -1 & 1 & 1 & -1 & -1 & 1 & -1 & -1 & -1 & & \\
$B_{2 u}$ & 1 & -1 & 1 & -1 & 1 & -1 & 1 & -1 & 1 & 1 & & \\
$E_{u}$ & 2 & 0 & -2 & 0 & 0 & -2 & 0 & 2 & 0 & 0 & $(x, y)$ & \\
\hline
\end{tabular}


Table 2. Correlation table for the D4h point group and its subgroups. (Fateley, W.G., Golish, F.R., McDevitt, N.T., Bentley, F.F. Infrared and Raman Selection Rules for Molecular and Lattice Vibrations: The Correlation Methods, Wiley-Interscience: New York, 1972; Salthouse, J.A. Ware, M.J., Point Group Character Tables, University Press: Cambridge, 1972.)

\begin{tabular}{|c|c|c|c|c|c|c|c|c|c|c|c|c|c|}
\hline \multirow[b]{3}{*}{$\begin{array}{l}A_{1 g} \\
A_{2 g} \\
B_{1 g} \\
B_{2 g} \\
E_{g}\end{array}$} & \multirow{3}{*}{$\begin{array}{l}\mathbf{D}_{4} \\
\mathrm{~A}_{1} \\
\mathrm{~A}_{2} \\
\mathrm{~B}_{1} \\
\mathrm{~B}_{2} \\
\mathrm{E}\end{array}$} & \multicolumn{2}{|c|}{$D_{2 h}$} & \multicolumn{2}{|c|}{$D_{2 d}$} & \multirow{3}{*}{$\begin{array}{l}C_{4 h} \\
A_{g} \\
A_{g} \\
B_{g} \\
B_{g} \\
E_{g}\end{array}$} & \multirow{3}{*}{$\begin{array}{l}\mathbf{C}_{4 \mathrm{~V}} \\
\mathrm{~A}_{1} \\
\mathrm{~A}_{2} \\
\mathrm{~B}_{1} \\
\mathrm{~B}_{2} \\
\mathrm{E}\end{array}$} & \multirow{3}{*}{$\begin{array}{l}\mathrm{C}_{4} \\
\\
\mathrm{~A} \\
\mathrm{~A} \\
\mathrm{~B} \\
\mathrm{~B} \\
\mathrm{E}\end{array}$} & \multirow{3}{*}{$\begin{array}{l}\mathbf{S}_{4} \\
\\
A \\
\text { A } \\
\text { B } \\
\text { B } \\
\text { E }\end{array}$} & \multicolumn{2}{|c|}{$\mathrm{D}_{2}$} & \multicolumn{2}{|c|}{$C_{2 v}$} \\
\hline & & $\mathrm{C}_{2}^{\prime}$ & $\mathrm{C}_{2}$ & $\mathrm{C}_{2}^{\prime}$ & $\mathrm{C}_{2}^{\prime \prime}$ & & & & & $\mathrm{C}_{2}^{\prime}$ & $\mathrm{C}_{2}^{n}$ & $\mathrm{C}_{2}, \sigma_{\mathrm{v}}$ & $\mathrm{C}_{2}, \sigma_{d}$ \\
\hline & & $\begin{array}{l}A_{g} \\
B_{1 g} \\
A_{g} \\
B_{1 g} \\
B_{2 g}+B_{3 g}\end{array}$ & $\begin{array}{l}A_{g} \\
B_{1 g} \\
B_{1 g} \\
A_{g} \\
B_{2 g}+B_{3 g}\end{array}$ & $\begin{array}{l}A_{1} \\
A_{2} \\
B_{1} \\
B_{2} \\
E\end{array}$ & $\begin{array}{l}A_{1} \\
A_{2} \\
B_{2} \\
B_{1} \\
E\end{array}$ & & & & & $\begin{array}{l}A \\
B_{1} \\
A \\
B_{1} \\
B_{2}+B_{3}\end{array}$ & $\begin{array}{l}A \\
B_{1} \\
B_{1} \\
A \\
B_{2}+B_{3}\end{array}$ & $\begin{array}{l}A_{1} \\
A_{2} \\
A_{1} \\
A_{2} \\
B_{1}+B_{2}\end{array}$ & $\begin{array}{l}A_{1} \\
A_{2} \\
A_{2} \\
A_{1} \\
B_{1}+B_{2}\end{array}$ \\
\hline $\begin{array}{l}A_{1 u} \\
A_{2 u} \\
B_{1 u} \\
B_{2 u} \\
E_{u} \\
\end{array}$ & $\begin{array}{l}A_{1} \\
A_{2} \\
B_{1} \\
B_{2} \\
E\end{array}$ & $\begin{array}{l}A_{u} \\
B_{1 u} \\
A_{u} \\
B_{1 u} \\
B_{2 u}+B_{3 u}\end{array}$ & $\begin{array}{l}A_{u} \\
B_{1 u} \\
B_{1 u} \\
A_{u} \\
B_{2 u}+B_{3 u}\end{array}$ & $\begin{array}{l}B_{1} \\
B_{2} \\
A_{1} \\
A_{2} \\
E\end{array}$ & $\begin{array}{l}B_{1} \\
B_{2} \\
A_{2} \\
A_{1} \\
E\end{array}$ & $\begin{array}{l}A_{u} \\
A_{u} \\
B_{u} \\
B_{u} \\
E_{u}\end{array}$ & $\begin{array}{l}A_{2} \\
A_{1} \\
B_{2} \\
B_{1} \\
E\end{array}$ & $\begin{array}{l}A \\
A \\
B \\
B \\
E\end{array}$ & $\begin{array}{l}B \\
B \\
A \\
A \\
E\end{array}$ & $\begin{array}{l}A \\
B_{1} \\
A \\
B_{1} \\
B_{2}+B_{3}\end{array}$ & $\begin{array}{l}\mathrm{A} \\
\mathrm{B}_{1} \\
\mathrm{~B}_{1} \\
\mathrm{~A} \\
\mathrm{~B}_{2}+\mathrm{B}_{3}\end{array}$ & $\begin{array}{l}A_{2} \\
A_{1} \\
A_{2} \\
A_{1} \\
B_{1}+B_{2}\end{array}$ & $\begin{array}{l}A_{2} \\
A_{1} \\
A_{1} \\
A_{2} \\
B_{1}+B_{2}\end{array}$ \\
\hline
\end{tabular}

\begin{tabular}{|c|c|c|c|c|c|c|c|c|c|c|c|c|}
\hline$\partial_{4 h}$ & \multicolumn{2}{|c|}{$C_{2 v}$} & \multicolumn{3}{|c|}{$\mathrm{C}_{4 \mathrm{~h}}$} & \multicolumn{3}{|c|}{$\mathrm{C}_{2}$} & \multicolumn{3}{|c|}{$C_{s}$} & $C_{i}$ \\
\hline & $\mathrm{C}_{2}^{\prime}$ & $\mathrm{C}_{2}{ }_{2}$ & $\mathrm{C}_{2}$ & $\mathrm{C}_{2}$ & $C_{2}^{\prime \prime}$ & $\mathrm{C}_{2}$ & $\mathrm{C}_{2}^{\prime}$ & $\mathrm{C}_{2}^{n}$ & $\sigma_{n}$ & $\sigma_{\mathrm{v}}$ & $\sigma_{d}$ & \\
\hline $\begin{array}{l}A_{1 g} \\
A_{2 g} \\
B_{19} \\
B_{2 g} \\
E_{g}\end{array}$ & $\begin{array}{l}A_{1} \\
B_{1} \\
A_{1} \\
B_{1} \\
A_{2}+B_{2}\end{array}$ & $\begin{array}{l}A_{1} \\
B_{1} \\
B_{1} \\
A_{1} \\
A_{2}+B_{2}\end{array}$ & $\begin{array}{l}A_{g} \\
A_{g} \\
A_{g} \\
A_{g} \\
2 B_{g}\end{array}$ & $\begin{array}{l}A_{g} \\
B_{g} \\
A_{g} \\
B_{g} \\
A_{g}+B_{g}\end{array}$ & $\begin{array}{l}A_{g} \\
B_{g} \\
B_{g} \\
A_{g} \\
A_{g}+B_{g}\end{array}$ & $\begin{array}{l}A_{g} \\
A_{g} \\
B_{g} \\
B_{g} \\
2 B \\
\end{array}$ & $\begin{array}{l}A_{1} \\
A_{2} \\
B_{1} \\
B_{2} \\
A+B\end{array}$ & $\begin{array}{l}A \\
A \\
B \\
B \\
A+B\end{array}$ & $\begin{array}{l}A^{\prime} \\
A^{\prime} \\
A^{\prime} \\
A^{\prime} \\
2 A^{n}\end{array}$ & $\begin{array}{l}A^{\prime} \\
A^{\prime \prime} \\
A^{\prime} \\
A^{\prime \prime} \\
A^{\prime}+A^{n}\end{array}$ & $\begin{array}{l}A^{\prime} \\
A^{\prime \prime} \\
A^{\prime \prime} \\
A^{\prime} \\
A^{\prime}+A^{\prime \prime}\end{array}$ & $\begin{array}{l}A_{g} \\
A_{g} \\
A_{g} \\
A_{g} \\
2 A_{g}\end{array}$ \\
\hline $\begin{array}{l}\mathrm{A}_{1 \mathrm{u}} \\
\mathrm{A}_{2 u} \\
\mathrm{~B}_{1 \mathrm{u}} \\
\mathrm{B}_{2 \mathrm{u}} \\
\mathrm{E}_{u}\end{array}$ & $\begin{array}{l}A_{2} \\
B_{2} \\
A_{2} \\
B_{2} \\
A_{1}+B_{1}\end{array}$ & $\begin{array}{l}\mathrm{A}_{2} \\
\mathrm{~B}_{2} \\
\mathrm{~B}_{2} \\
\mathrm{~A}_{2} \\
\mathrm{~A}_{1}+\mathrm{B}_{1}\end{array}$ & $\begin{array}{l}A_{u} \\
A_{u} \\
A_{u} \\
A_{u} \\
2 B_{u}\end{array}$ & $\begin{array}{l}A_{u} \\
B_{u} \\
A_{u} \\
B_{u} \\
A_{u}+B_{u}\end{array}$ & $\begin{array}{l}A_{u} \\
B_{u} \\
B_{u} \\
A_{u} \\
A_{u}+B_{u}\end{array}$ & $\begin{array}{l}\mathrm{A} \\
2 \mathrm{~B}\end{array}$ & $\begin{array}{l}A \\
B \\
A \\
B \\
A+B\end{array}$ & $\begin{array}{l}A \\
B \\
B \\
A \\
A+B\end{array}$ & $\begin{array}{l}A^{\prime \prime} \\
A^{\prime \prime} \\
A^{\prime \prime} \\
A^{\prime \prime} \\
2 A^{\prime}\end{array}$ & $\begin{array}{l}A^{\prime \prime} \\
A^{\prime} \\
A^{\prime \prime} \\
A^{\prime} \\
A^{\prime}+A^{\prime \prime}\end{array}$ & $\begin{array}{l}A^{\prime \prime} \\
A^{\prime} \\
A^{\prime} \\
A^{\prime \prime} \\
A^{\prime}+A^{n}\end{array}$ & $\begin{array}{l}A_{u} \\
A_{u} \\
A_{u} \\
A_{u} \\
2 A_{u}\end{array}$ \\
\hline
\end{tabular}




\section{FIGURES}

Figure 1. Definitions of distance in Eq (2).

Figure 2. Vibronic structure in absorption spectra.

Figure 3. Typical metal porphyrin absorption spectrum illustrating the observed vibronic structure for the $Q$ transition. The intensity of the vibrational side band is partly a consequence of intensity borrowing from the strong $B$ transition. It is also influenced by distortion in the ground electronic state as quantified by normalcoordinate structural decomposition. 


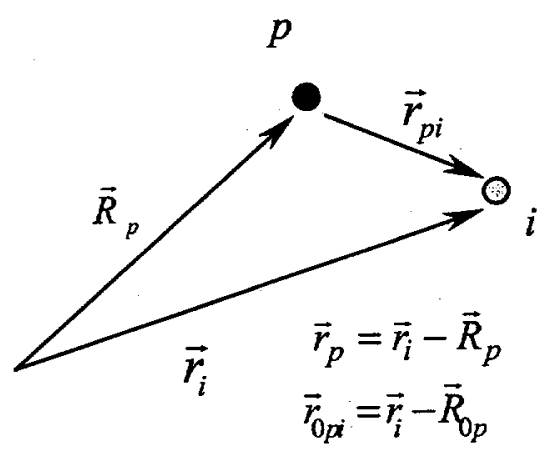

Figure 1. 
UV-visible

IR

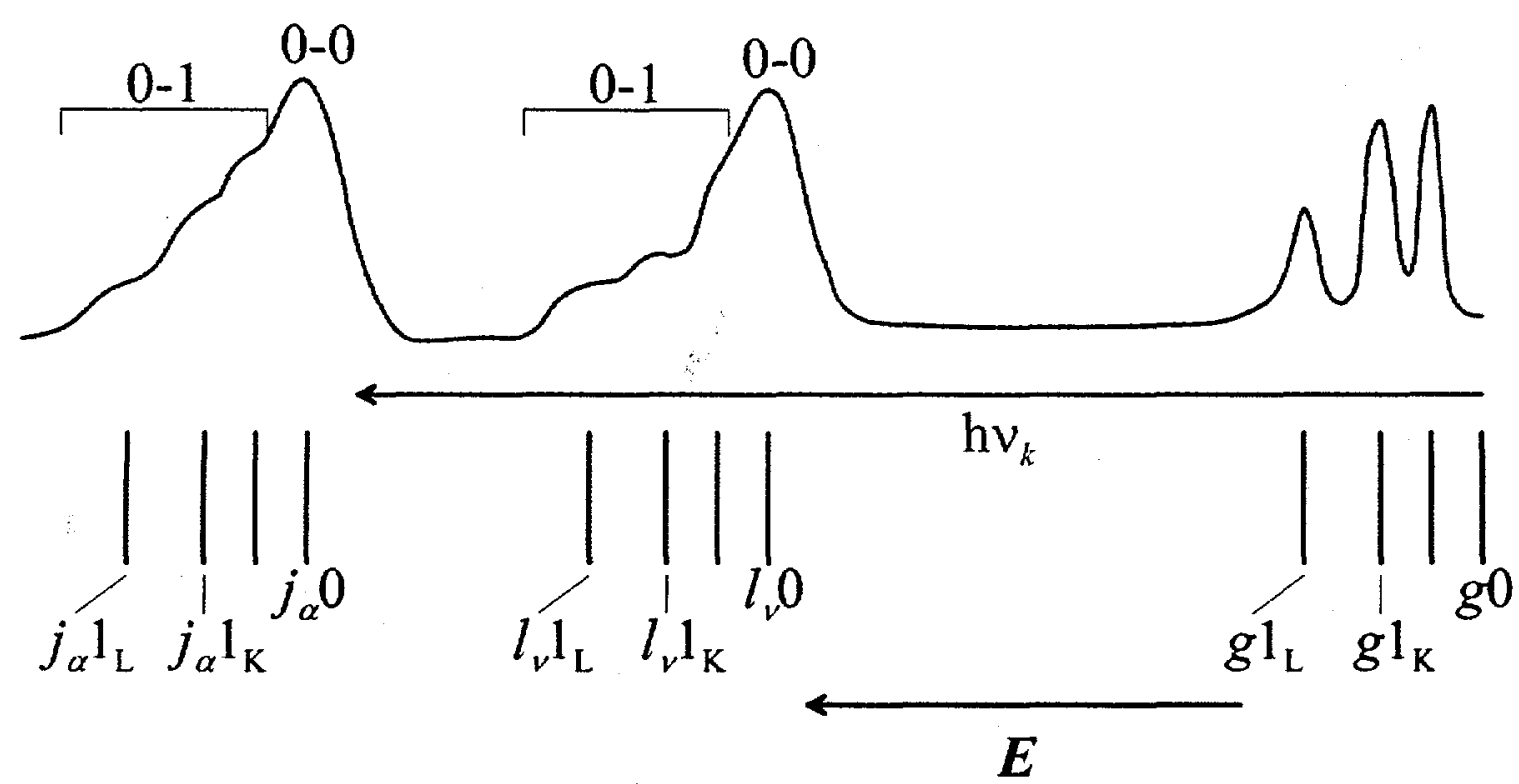

Figure 2. 


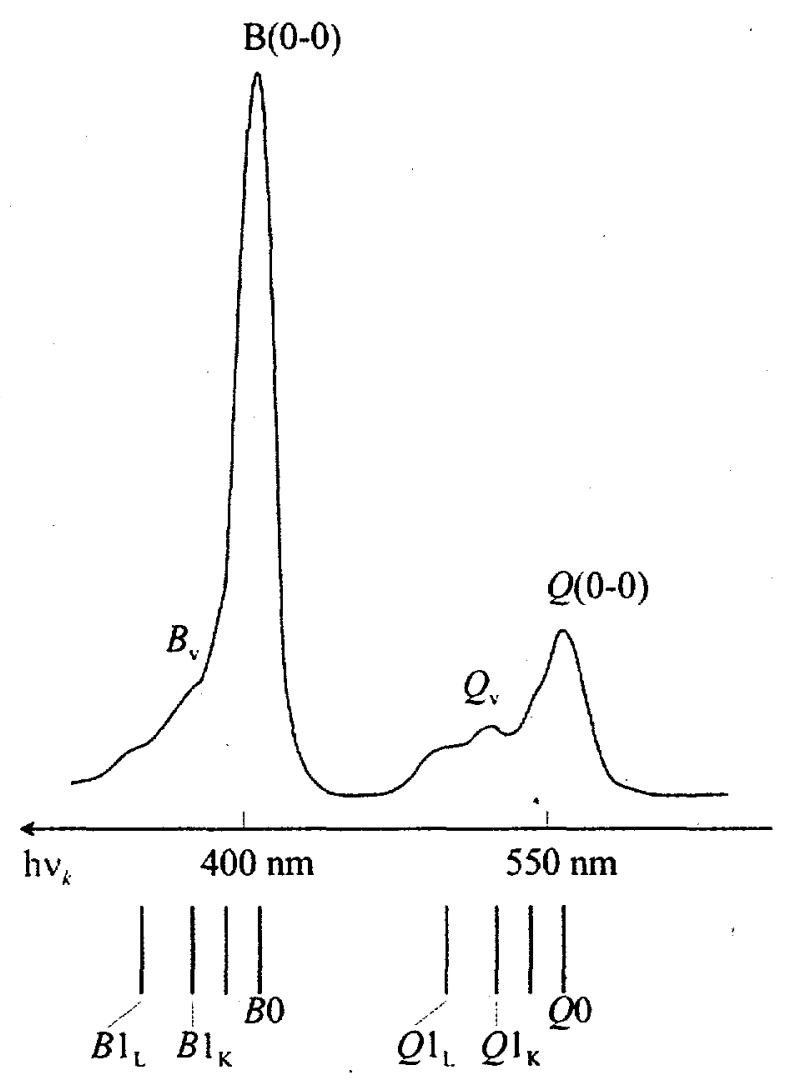

Figure 3. 